Review

\title{
Computer-Aided Diagnosis Systems for Brain Diseases in Magnetic Resonance Images
}

\author{
Hidetaka Arimura $^{1, *}$, Taiki Magome ${ }^{2}$, Yasuo Yamashita ${ }^{2}$ and Daisuke Yamamoto ${ }^{2}$ \\ 1 Faculty of Medical Sciences, Kyushu University, Fukuoka 812-8582, Japan \\ 2 Graduate School of Medical Sciences, Kyushu University, Fukuoka 812-8582, Japan \\ * Author to whom correspondence should be addressed. E-mail: arimura@shs.kyushu-u.ac.jp
}

Received: 27 March 2009; in revised form: 6 June 2009 / Accepted: 2 July 2009 /

Published: 10 July 2009

\begin{abstract}
This paper reviews the basics and recent researches of computer-aided diagnosis (CAD) systems for assisting neuroradiologists in detection of brain diseases, e.g., asymptomatic unruptured aneurysms, Alzheimer's disease, vascular dementia, and multiple sclerosis (MS), in magnetic resonance (MR) images. The CAD systems consist of image feature extraction based on image processing techniques and machine learning classifiers such as linear discriminant analysis, artificial neural networks, and support vector machines. We introduce useful examples of the CAD systems in the neuroradiology, and conclude with possibilities in the future of the CAD systems for brain diseases in MR images.
\end{abstract}

Keywords: Computer-Aided Diagnosis (CAD) system; brain diseases; Magnetic Resonance (MR) images; image processing techniques

\section{Introduction}

A number of institutions perform special medical checks for brain, known as "Brain Dock" in Japan, by using advanced imaging techniques like computed tomography (CT), magnetic resonance imaging (MRI) and positron emission CT (PET), because the Japanese are also keen in preventing various neurological conditions including stroke, dementia, etc. The purpose of the Brain Dock is to detect asymptomatic brain diseases in their early stages, e.g., asymptomatic lacunar infarction, unruptured intracranial aneurysms, dementia, and to prevent such cerebral disorders [1]. As for stroke, for instance, the asymptomatic lacunar infarction and unruptured intracranial aneurysms should be 
detected for the prevention of cerebral infarction and subarachnoid hemorrhage (SAH), respectively. Furthermore, dementing disorders such as Alzheimer's disease (AD) and vascular dementia are major public health problems in countries with greater longevity, such as in Japan, where the average life expectancy was 82 years in 2004 [2]. For the detection of the AD, neuroradiologists attempt to evaluate the degree of cerebral atrophy subjectively based on cortical thicknesses in the temporal lobe or parietal lobe, sizes of hippocampus and entorhinal cortex, and expansion of lateral ventricles. On the other hand, multiple sclerosis (MS) is one of the most serious neurological disorders, which develops in young adults. Multiple sclerosis is a progressive neurological disorder, which is caused by the structural damage of axons and their myelin sheathes in the central nervous system. The progression of the MS lesions shows considerable variability and MS lesions present temporal changes in shape, location, and area between patients and even in the same patient [3-6]. However, it would be very difficult to detect the lesions among a great number of images or a small number of patients out of many healthy people. It is possible for radiologists to miss the lesions of patients, because of their heavy workloads. Therefore, radiologists would need to experience a number of training sessions to detect the lesions of patients correctly and efficiently. In addition, the number of images, which neuroradiologists have to diagnose, has increased rapidly, because MRI has shifted from twodimensional (2D) imaging to 3D imaging, and the resolution as well as signal-to-noise ratio has become higher. Consequently, neuroradiologists have been demanding the new approach of "computer-aided diagnosis (CAD)" due to their heavy workloads in the field of neuroradiology.

CAD has become one of the major research subjects in medical imaging and diagnostic radiology. The basic concept of CAD was proposed by The University of Chicago, in the mid-1980s, whose idea it was to provide a computer output as a "second opinion" to assist radiologists in interpreting images, so that the accuracy and consistency of radiological diagnosis could be improved, and also the image reading time could be reduced [7-9]. Since then, a number of researchers have developed computeraided diagnostic CAD schemes for detection and classification of various kinds of abnormalities such as microcalcifications and masses in mammograms [10-13], pulmonary nodules and diffuse lung diseases in CT [14-18], and colonic polyps in CT colonography [19-22]. Besides, the clinical usefulness of CAD schemes has been studied by performing the receiver operating characteristic (ROC) analysis in experiments similar to clinical situations [10,16,23,24]. However, it has been said that it would be arduous to develop CAD systems using MRI, because the image quality of MR images including specific artifacts and image noise could vary with imaging sequences and patients.

In recent years, various kinds of CAD methods have been developed in the neuroradiology using MR images [25-31]. Radiologists expect that CAD systems can improve their diagnostic abilities based on synergistic effects between the computer's and radiologist's high abilities by using the information analysis including the medical images. For instance, Figure 1 shows an illustration of the output by a CAD system for detection of intracranial aneurysms in MR angiography (MRA) images. In this review paper, we describe the basics and the recent researches of CAD systems for brain diseases in the field of neuroradiology. In Section 2, the fundamental techniques used for CAD systems for neuroradiology are explained, including image processing and pattern recognition. Several application examples of CAD approaches are introduced in Section 3, including detection of abnormalities, e.g., aneurysms in MRA images, white matter hyperintensities (ischemic lesions and 
lacunar infarction), Alzheimer's disease, MS lesions, and classification of brain tumors in MR images. In Section 4, we conclude with possibilities in the future of CAD systems.

Figure 1. An illustration of the output by a CAD system for detection of intracranial aneurysms in MRA images [25,26].

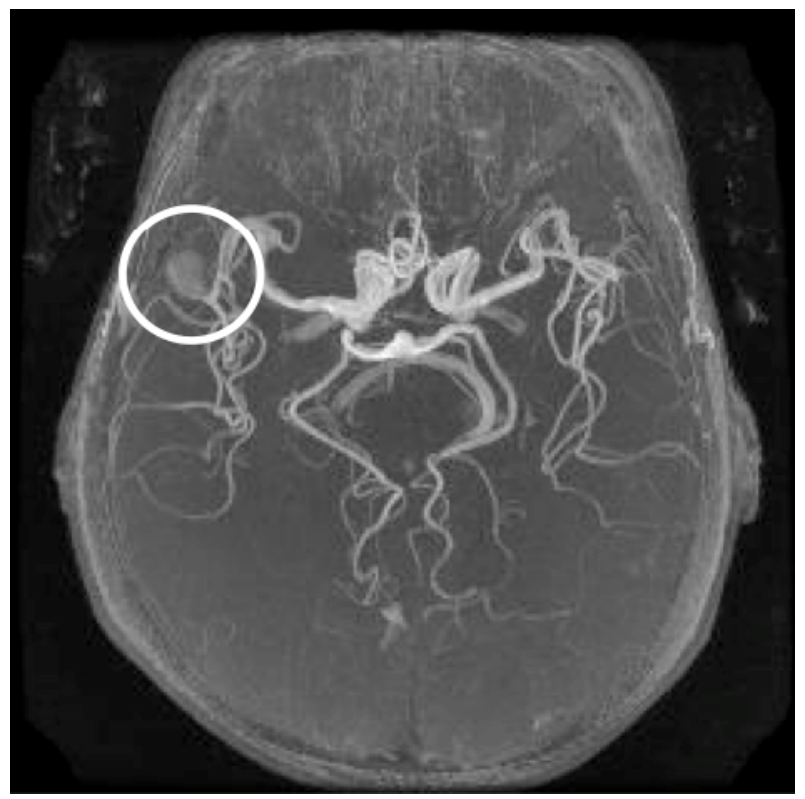

\section{Basics of CAD systems}

\subsection{CAD systems and pattern recognition}

In general, radiologists carefully look into medical images, and diagnose a patient's disease by obtaining useful information and interpreting medical images with their experience, knowledge, and wisdom. For the purpose of attaining a correct diagnosis, radiologists need to learn and archive a huge number of cases with various types of diseases in their own mind according to their specialty. A good radiologist does not need to have a particularly good eye, but rather a sensible ability to identify precisely what he or she is looking at when looking at medical images. Thus, it can be said that good radiologists have high and flexible abilities of learning and pattern recognition. Since CAD systems provide radiologists with a "second opinion" for assisting them in the diagnosis of patients' diseases, it entails the CAD system to have similar abilities as the radiologists in terms of learning and pattern recognition, even though good CAD systems cannot reach radiologists' high diagnostic abilities. Consequently, machine learning and pattern recognition techniques are substantially important in developing CAD systems.

Pattern recognition is the act of extracting features from some objects in raw data and making a decision based on the classifier output such as classifying each object into one of the possible categories of various patterns [32]. In general, there are two types of CAD systems. One CAD system is for classifying all candidates into abnormal and normal candidates such as intracranial aneurysms or white matter hyperintensities in MR images, i.e., two-class categorization system. The other is a CAD system for classification of unknown cases into several types of abnormalities, which are more than two, i.e., multi-class categorization system. For instance, one CAD system would be for the 
classification of brain tumors into high grade glioma, metastatic brain tumor, low grade glioma, malignant lymphoma, etc.

Figure 2 shows the difference between a general pattern recognition system and a CAD system. Basically, both systems consist of preprocessing, feature extraction, and classification. However, there is one difference between the two systems. The CAD systems would suggest a "second opinion" to radiologists, not an output "decision," which should be made by the radiologists using the CAD systems. Nevertheless, algorithms of major CAD systems have been developed by using techniques and theories developed in the pattern recognition field, and thus we believe that a computer-aided diagnosis is academically included as one of the pattern recognition fields. The techniques of the CAD systems on preprocessing, feature extraction, and machine learning classification are described in the subsequent sections.

Figure 2. Difference between (a) a general pattern recognition system and (b) a computeraided diagnosis system. Note that the scheme of Feature extraction step is described in Figure 3.

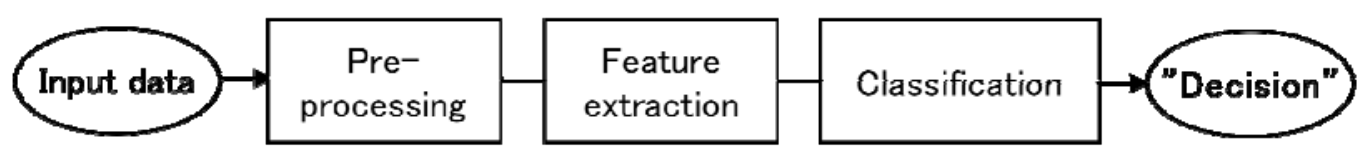

(a)

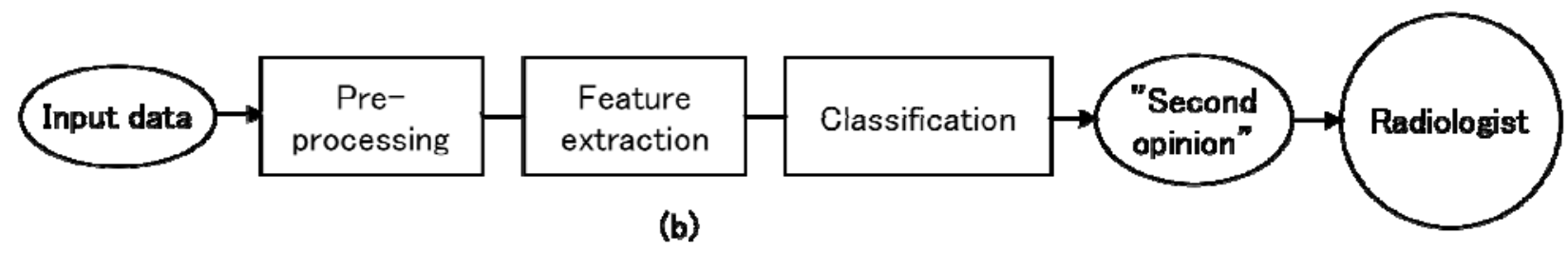

\subsection{Preprocessing}

Preprocessing of images is essential for reducing the complexity and computation time of the CAD algorithms. Since the image quality of the MR images depends on patients and/or MR imaging sequences, there is much variability in the pixel value range, noise level, and background level. Therefore, the normalization of pixel values, some smoothing filters for reducing noise, background correction, etc., should be performed prior to input into the feature extraction. For instance, in accurate segmentation of anatomical regions such as white matter and gray matter regions, the edge preserving smoothing (EPS) filters [33,34] would be useful for preserving the boundary contrast between anatomical regions as well as for reducing the noise.

As for another important preprocessing, the region of interest (ROI) for searching abnormalities should be segmented for reducing the computation time, taking into account anatomical regions, where abnormalities could be located. For example, a search region was determined by dilation of major vessels in a CAD system for detection of aneurysms in MRA [25]. In a CAD system for detection of white matter hyperintensities or MS lesions in MR images, the brain regions were segmented as search regions by using basic statistical quantities of tissue peaks in a pixel-value histogram in the original MR images. Also, the spatial position corrections for the head displacement and inclination are important for determination of a reference point or an origin in each patient. Furthermore, the 
coordinate system in the brain such as Talairach coordinates [35] is often necessary for considering the functional area such as Brodmann area.

\subsection{Feature extraction}

The feature extraction is one of the main steps for CAD systems, but there is no "royal road" to extracting features in the CAD field as well as the pattern recognition field. That is because each method for feature extraction depends on each object (lesion) in each medical image. Consequently, a number of feature extraction methods have been developed for specific lesions. Figure 3 shows a scheme of feature extraction in a CAD system. At first in preparation for easily detecting initial lesion candidates at the next step, the object should be enhanced by some methods, as shown in Figures 4 and 5. Then, the candidate regions are extracted by some segmentation methods. Finally, image features are calculated as inputs for a classifier. The details of each step are described in the following subsections.

Figure 3. Scheme of feature extraction in a CAD system.

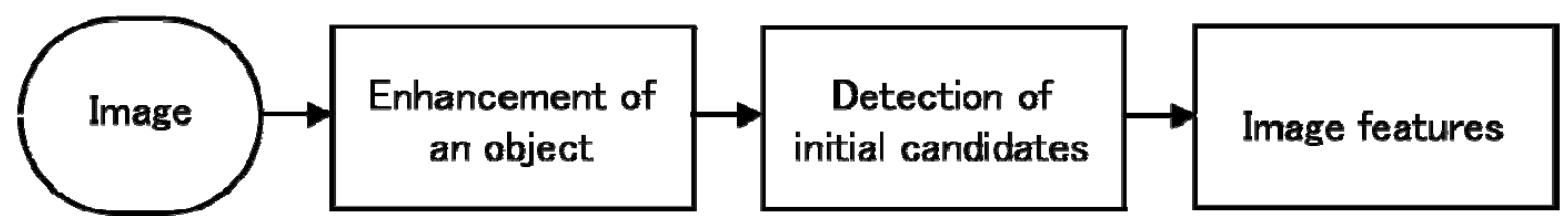

\subsubsection{Image enhancement of an object}

If the gray-level spatial distribution within the object is known, the enhancement filter could be developed. For example, small intracranial aneurysms, which are imaged by MR scanners, could be enhanced by using an enhancement filter for a 3D Gaussian shape, because the shapes of the aneurysms are similar to 3D Gaussian function. Therefore, several enhancement filters for specific lesions have been developed in the CAD field [36-42]. Giger et al. developed a difference image technique for the detection of lung nodules in radiographs, based on the subtraction of the objectsuppressed image using a ring filter from the object-enhanced image using an object-matched filter $[36,43]$. Arimura et al. applied the difference image technique to the automated detection of lung nodules in low-dose CT [44]. Yamamoto et al. developed a morphological filter (Quoit filter) to extract the isolated nodules, and they applied the filter to automatic detection of lung nodules in CT images [37]. Sato et al. proposed a three-dimensional (3D) filters based on a combination of the eigenvalues of the Hessian matrix of the 3D image for enhancement of specific 3D structures, which are line-like, sheet-like, and blob-like structures corresponding to items such as cerebral vessels, vessel walls, and aneurysms, respectively [38,39]. By using the same theory as Sato et al., Li et al. developed selective enhancement filters for $\operatorname{dot}(=\mathrm{blob})$, line, and plane (=sheet) objects [40]. In the neuroradiological CAD field, Arimura et al. applied selective enhancement filters for detection of unruptured intracranial aneurysms in MRA images [25, 26]. Figure 4 shows the effect of a dotenhancement filter for an MRA image with an unruptured intracranial aneurysm. The balloon-like aneurysms are well enhanced by the dot-enhancement filter. Hayashi et al. proposed a curvature-based 
enhanced display system for detecting cerebral aneurysms in MR angiography [41]. Yamamoto and Arimura et al. proposed a simple method for enhancing MS lesions in 2D fluid-attenuated-inversionrecovery (FLAIR) images by subtracting the background image approximated by the first order polynomial in a brain parenchymal region [30,31]. Figure 5 shows the effect of the background subtraction image on enhancement of MS lesions. Relatively low-contrast MS lesions are well enhanced by suppressing the background in the brain parenchyma. However, one of the problems caused by such kinds of enhancement filters is that the filter induces enhancement of the lesion-like normal structures as well. Consequently, we have to reduce numerous false positives by obtaining a number of image features of the candidate regions enhanced by the filter. To overcome the issue, Suzuki et al. attempted to develop a supervised filter for enhancement of lesions by use of a massivetraining artificial neural network (MTANN) in a CAD scheme for detection of lung nodules in CT [42]. The MTANN filter is trained with actual lesions in CT images to enhance the actual patterns of the lesions, not lesion-like normal structures. This supervised filter could be promising for developing CAD systems in the neuroradiological field.

Figure 4. Effect of a dot-enhancement filter for a MRA image with an unruptured intracranial aneurysm: (a) an original MRA image and (b) a dot-enhanced image $[25,26]$.

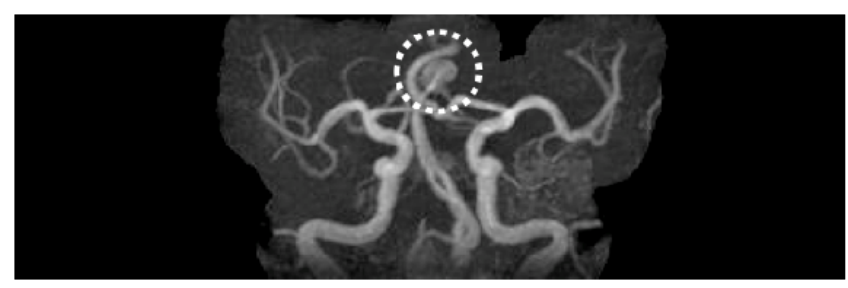

(a)

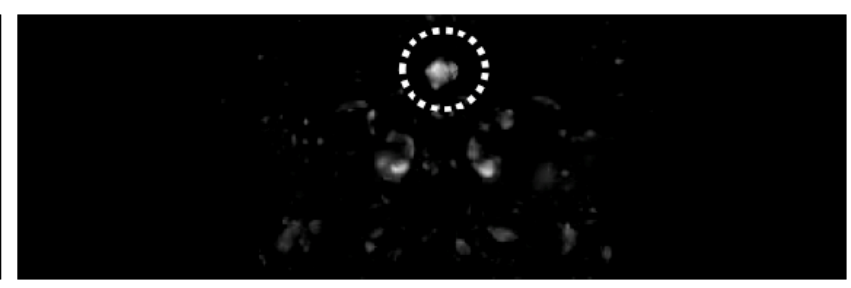

(b)

Figure 5. Illustrations of (a) an original FLAIR image, and (b) its corresponding subtraction image between the FLAIR image and a background image approximated by the first order polynomial in a brain region [30,31].

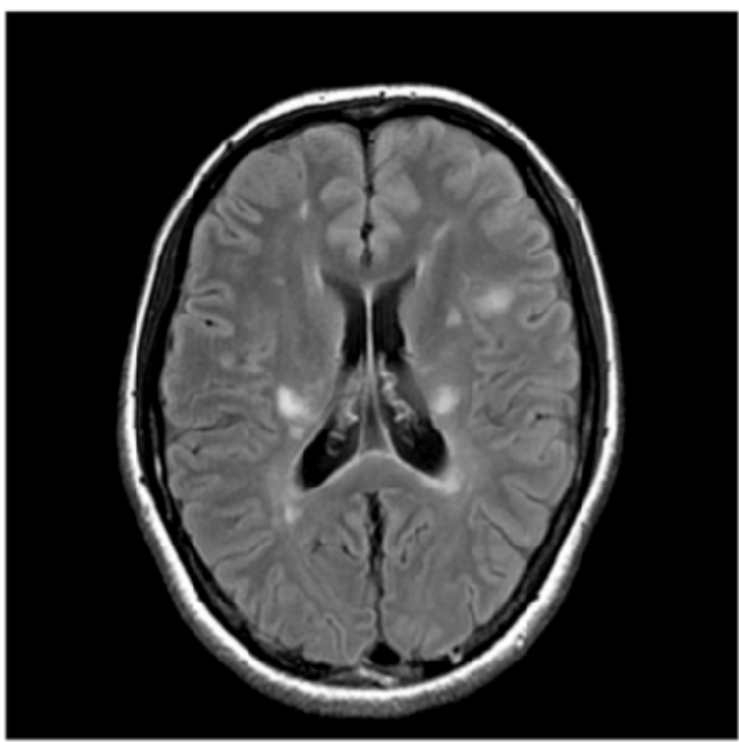

(a)

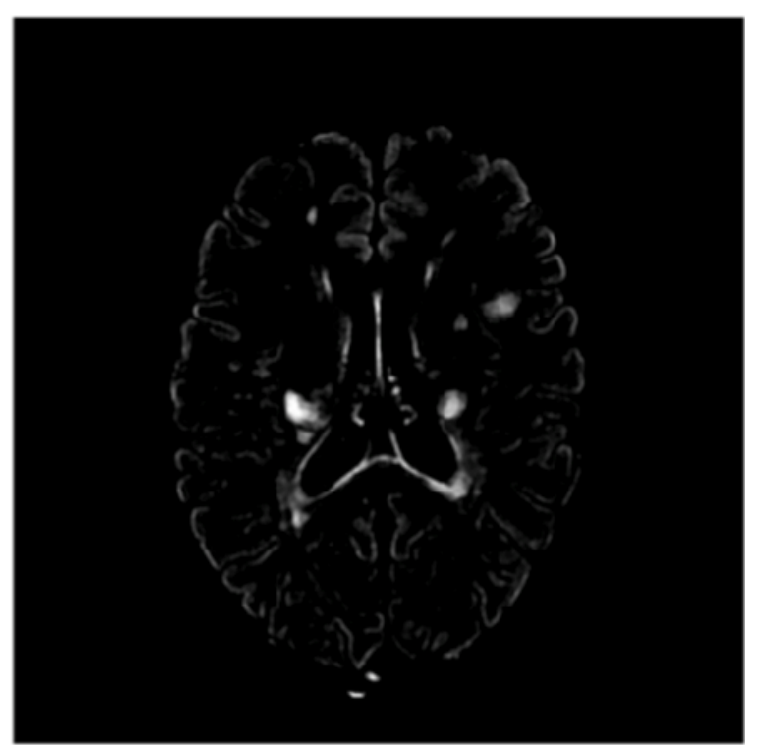

(b) 


\subsubsection{Detection of initial lesion candidates}

Although a number of methods for initial detection of lesions have been developed in the CAD field, basic simple thresholding techniques for outputs of enhancement filters or pixel values in the object-enhanced images have been employed for initial identification of lesions. As a unique method, Lee et al. developed an initial detection method of lung nodules using two template matching techniques based on a genetic algorithm and cross-correlation coefficient [45]. Furthermore, although it may not be directly related to the initial lesion detection, a method based on probabilistic atlases has been developed for determination of some anatomical regions such as cerebral cortical regions [46,47]. Probabilistic atlases could store information on anatomic and functional variability in a population. We have applied a multiple-gray level thresholding technique developed by Giger et al. [21,43] to the neuroradiological CAD field, for detection of initial aneurysm candidates, white matter hyperintensities (WMHs) and MS candidates in MR images as the points with local maximum pixel values in candidate regions [25,26,28,30,31]. Figure 6 shows a FLAIR image including initial MS candidate points (white dots) obtained by the multiple gray level thresholding technique, and a brain image with initial candidate regions (white solid lines) segmented by the region growing technique $[30,31]$.

Figure 6. Illustrations of (a) an original FLAIR image, (b) initial MS candidate points (white dots) obtained by the multiple gray level thresholding technique, and (c) initial candidate regions (white solid lines) segmented by the region growing technique [30,31].

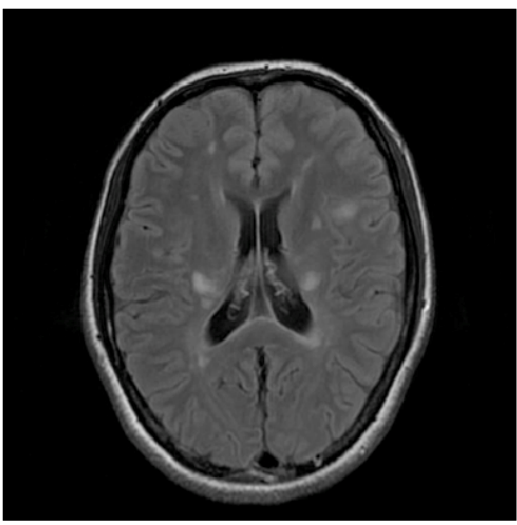

(a)

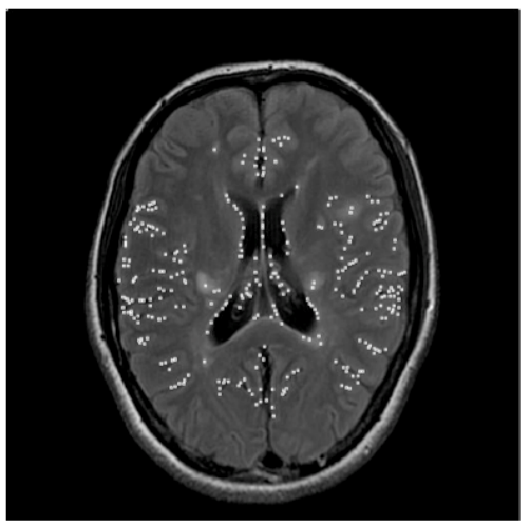

(b)

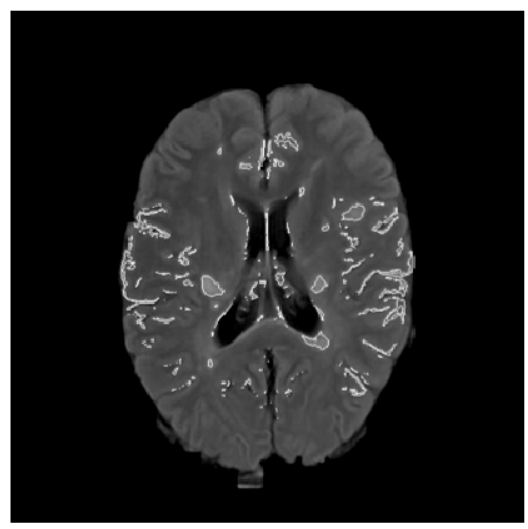

(c)

A number of segmentation methods have been proposed for extraction of lesion candidate regions or target anatomical regions, followed by image feature extraction. Major segmentation methods are based on region growing techniques [25,48], watershed algorithm [49], snake algorithm [50], and level set method [51]. In the image processing field of neuroradiology, the level set methods have been widely used for segmentation of anatomical regions such as brain parenchyma, white matter, and cerebral cortical regions [51-54]. First, a level set function $\phi$ was determined as a signed distance function from the contour of the initial white matter regions, which was the zero level in the level set function. Second, the level set function $\phi$ was solved according to the following partial differential equation: 


$$
\frac{\partial \phi}{\partial t}+F|\nabla \phi|=0
$$

where $t$ is the time and $F$ is the speed function. While updating the level set function, the zero level set $\phi=0$ moves according to the speed function in the higher dimensional level set function. Here, the zero level set is called as "moving front". Finally, the update of the level set function was stopped if some conditions are satisfied. Figures 7(a) and 7(b) show the illustrations of an original T2-weighted image and white matter regions segmented by a level set method, respectively [55].

Figure 7. Illustrations of: (a) an original T2-weighted image and (b) white matter regions segmented by a level set method [55].

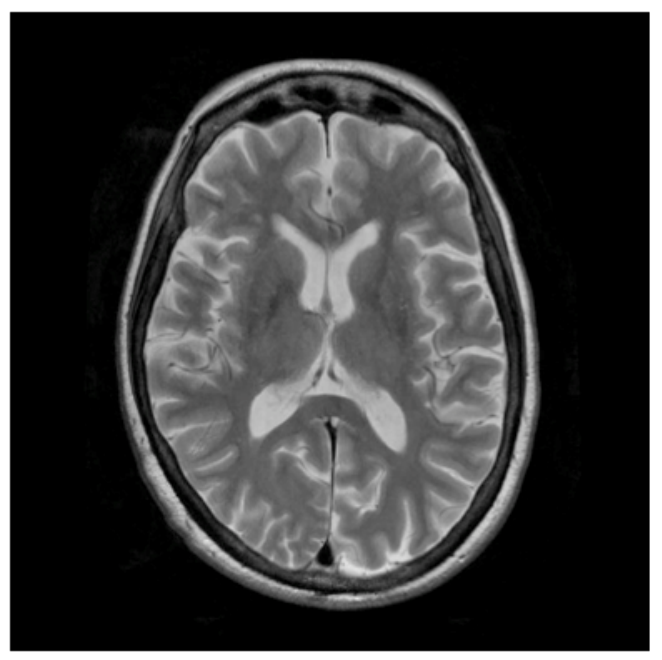

(a)

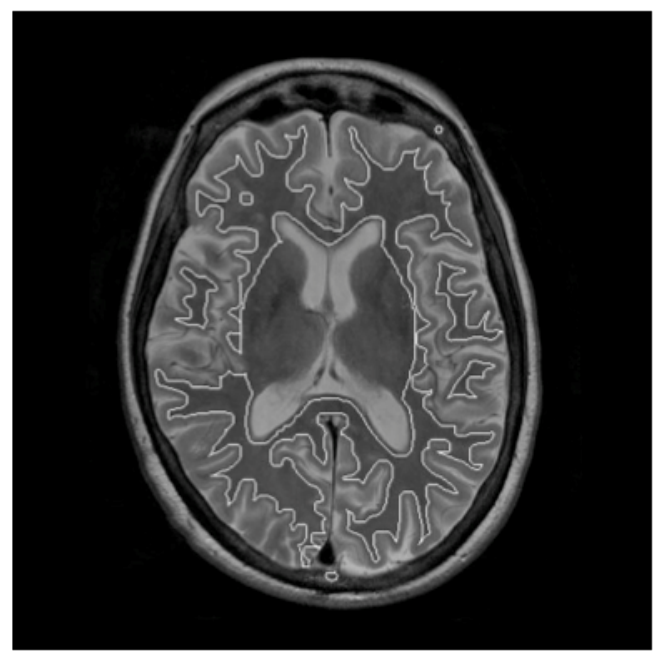

(b)

\subsubsection{Image feature extraction}

The goal of the feature extraction is to characterize an object to be recognized by measurements whose values are very similar to those for objects in the same category, but very different from those for objects in different categories [32]. The accuracy of the subsequent classification would depend on the variability in the feature values for objects in the same category (intraclass variability) relative to the difference between feature values for objects in different categories (interclass variability). In addition, features should be invariant to irrelevant transformations of the inputs such as translation, rotation, and scale [32]. However, the problem is how to determine the image features to characterize the objects. Let us consider characterizing MS lesions as a simple example. According to Figure 6, the shapes of the MS lesions look like circles or ellipsoids, and the MS lesions seem to be located near to the lateral ventricles or within white matter regions. However, a majority of false positives would be very small and linear, and be located far from the center of the brain or in gray matter regions. Therefore, as image features to characterize MS lesions, we chose the circularity and the distance between centroids of each candidate and a brain region, which are invariant to translation, rotation, and scale. Note that the distance should be normalized by patient-specific values such as the anteriorposterior distance of each brain region. Figures 8(a) and (b) show the relationships between the circularity and the distance between two centroids of each candidate and a brain region, for true MS regions and false positive regions, respectively [30,31]. These relationships back up the assumption 
derived from our observation in Figure 6. Many image features have been designed for characterizing specific lesions or diseases in the neuroradiological CAD system [25-31,41].

Effective features for detecting specific objects (lesions) should be selected by discussing with radiologists. By taking sufficient time and a careful look at images, CAD researchers should learn how the lesions look like. This activity would be to determine a model for the object such as a spherical shape for aneurysms. From the point of view of reducing false positives, several models for false positives should be determined such as a cylinder shape for vessel. Then, many features based on the models can be chosen such as effective diameter, sphericity, irregularity, slenderness.

Figure 8. Relationships between the distance between centroids of each candidate and a brain and circularity for (a) true positive regions and (b) false positive regions [30,31]. The dotted lines indicate a threshold value of 0.6 for dividing all MS candidate regions into two groups with higher and lower circularities.

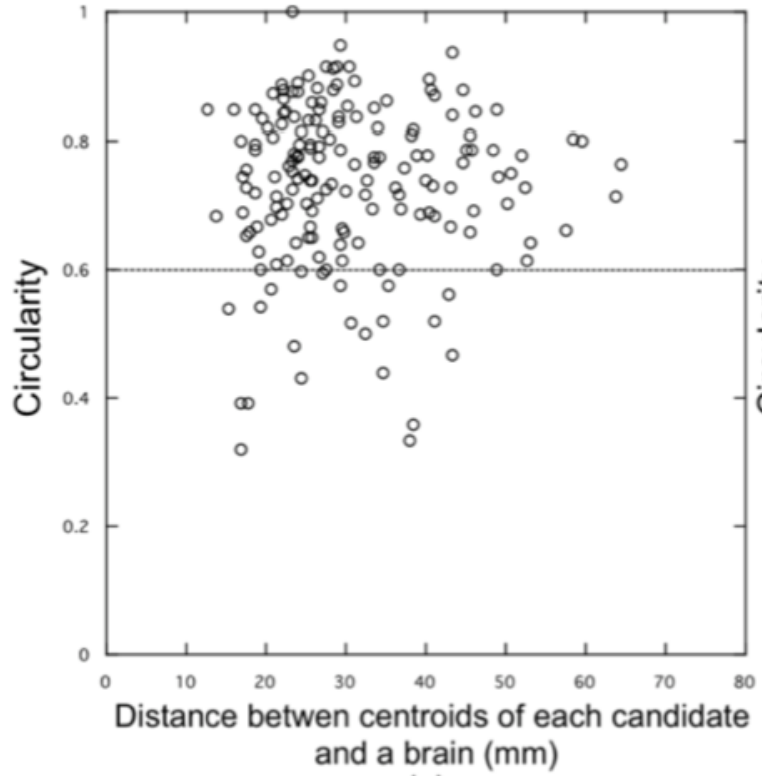

(a)

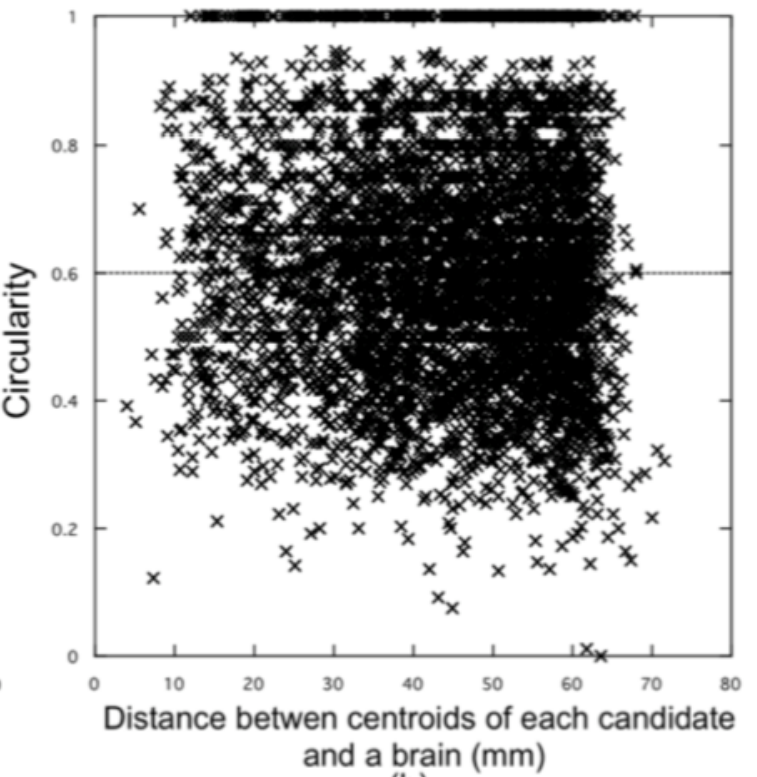

(b)

\subsection{Classification process}

In the classification process for CAD systems, the feature vector of each lesion candidate provided by a feature extractor is used for assigning the candidate to one of the possible categories (e.g. lesion or normal tissue) according to the output of a classifier. Prior to the practical test, the classifier should be designed by inputting feature values from training cases, and teaching the classifier true category labels (e.g., one or zero). This learning method is called, "supervised learning." In general, the unsupervised learning method, which does not need training data, has not been adopted so often in the CAD research fields, because the practical CAD systems should be "educated" or trained based on the clinical truth or evidence.

In order to improve generalization and robustness of a CAD system, the CAD system should be designed using a sufficient number of training cases. However, if a CAD system is trained by a small number of cases, the system may allow perfect classification of the training cases, but it is not likely to 
correctly classify unknown cases. This situation is known as overtraining. According to Qaing Li's report [56], the generalization accuracy (higher than 80\%) of a CAD system saturated from 150 test cases using a leave-one-out test method. Moreover, we should consider the curse of dimensionality, which causes the problem that classification performances of the CAD systems could deteriorate if the ratio of the number of training cases to that of features used for the classifier is relatively small [57]. A ratio of more than 5 or 10 would be better for avoiding the curse of dimensionality [57].

Major machine learning classifiers, which have been used for CAD systems, are linear discriminant analysis (LDA), quadratic discriminant analysis (QDA), artificial neural network (ANN), support vector machine (SVM), Bayesian-based classifier, etc. In a CAD system for Alzheimer's disease (AD) from Arimura et al. [27], AD cases were identified by using an SVM classifier, which was trained by atrophic image features (e.g., cortical thickness and cerebrospinal fluid (CSF) volume) of AD and non$\mathrm{AD}$ cases, and then an unknown case was classified into either AD or non-AD category based on an SVM model. The reasons why we employed the SVM as a classifier were high generalization ability, avoiding local minimum traps, and overcoming the curse of dimensionality [58, 59].

In the CAD system for classification of cerebral tumors, the ANN was designed to differentiate among 4 categories of tumors (high-grade gliomas, low-grade gliomas, metastases, and malignant lymphomas) with use of 2 clinical parameters (age and history of malignant tumor) and 13 radiologic findings (e.g., location, signal intensity on T2-wieghted images) in MR images [60]. Figure 9 shows an ANN classifier for differentiating cerebral tumors into 4 categories, which was composed of 15 input units and 4 output units.

Figure 9. An ANN classifier for differentiating cerebral tumors into 4 categories, which was composed of 15 input units and 4 output units [60].

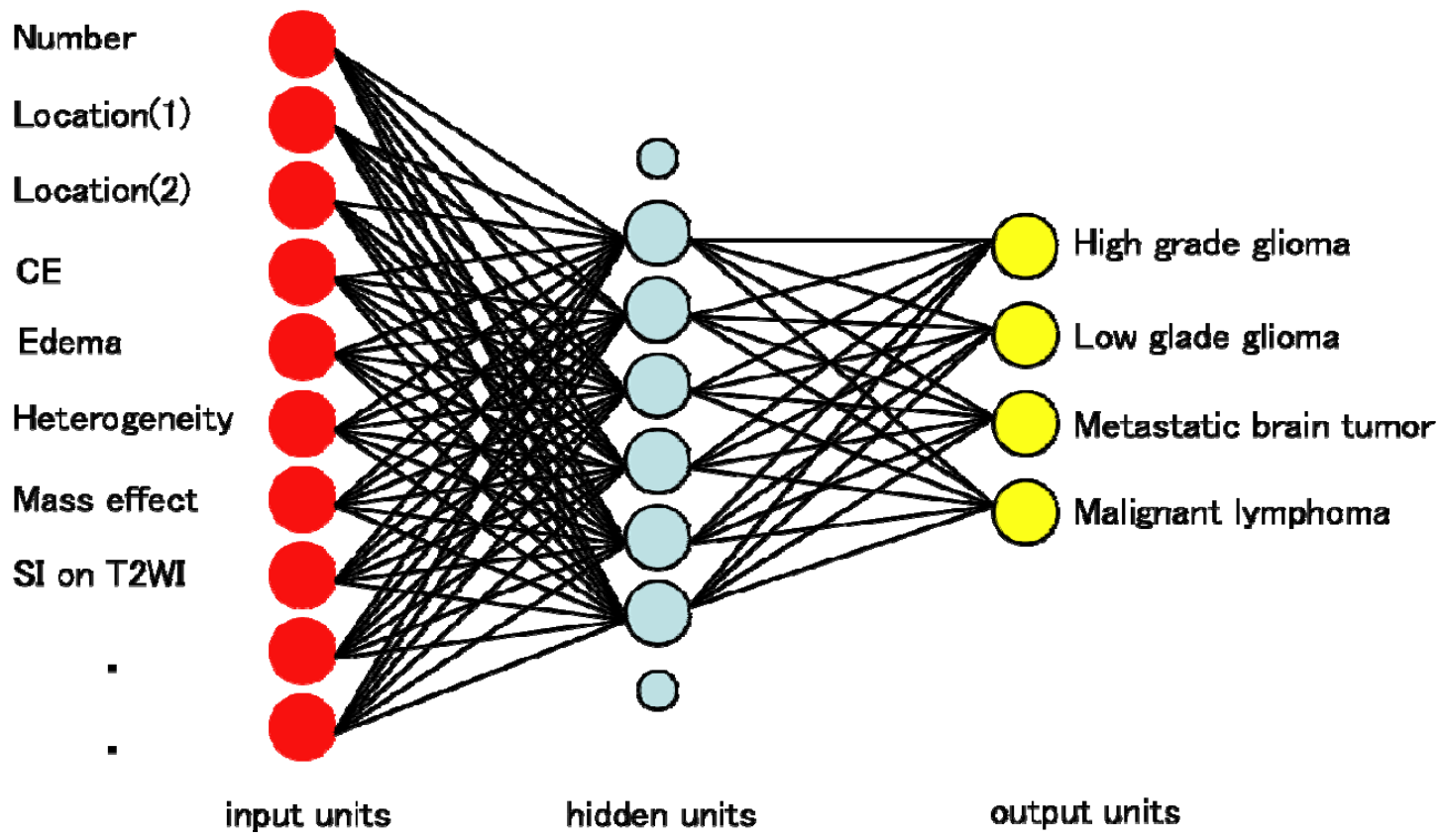


In many CAD systems, at first, simple rule-based methods have been used for reducing "obvious false positive outliers", and then the subsequent machine learning classifier for removing various kinds of many false positives including "fake positives", i.e., lesion-like false positives. The rules for removing false positive outliers are based on some simple threshold values, such as the minimum and maximum threshold values determined by a mean value \pm a few times standard deviation of the feature values of all true positive regions [30,31].

\subsection{Evaluation of $C A D$ systems}

The performance of CAD systems is evaluated by similar test methods to the general pattern recognition systems. The purpose of the test is to assess the generalization and robustness of the systems. The major test methods are resubstitution test, k-fold cross-validation test, and bootstrap method [57]. The resubstition test would be an initial test for the CAD system, where the training data is the same as the test data. In the k-fold cross-validation test, all lesion candidates or cases are randomly split into $\mathrm{k}$ datasets. The $\mathrm{k}-1$ datasets are used for training of the CAD system, and one dataset is used for testing. The training and testing are repeated by $\mathrm{k}$ times. When the number of $\mathrm{k}$ is equal to the number of candidates or cases, we call the test a leave-one-out test. The leave-one-out 'by patient' test method is often used in the CAD field. All lesion candidates except for the candidates obtained from the same patient were used for training, and the one candidate left out was used for testing with the CAD system. However, either large bias or large variance could be included in the cross-validation test methods. To reduce the bias or variance, the bootstrap method has been used [61], where a number of fake data sets of the same size were generated as the given training set, and the data sets were used for estimation of pattern recognition systems' performances [57].

Prior to the evaluation test, the lesion candidates should be separated into the true positives and false positives by using a criterion based on "truth". For instance, in the CAD system for detection of intracranial aneurysms, we used a criterion such that an aneurysm was considered correctly detected if the pixel with the maximum voxel value in the candidate region was located within the diameter of the aneurysm, which was measured by neuroradiologists, for aneurysms smaller than $7.0 \mathrm{~mm}$, and within the diameter of $7.0 \mathrm{~mm}$ for aneurysms larger than $7.0 \mathrm{~mm}$ [25]. Another example of a criterion is that if the centroid of a candidate region was in a "truth" region determined by neuroradiologists, the candidate region would be classified into a true positive [28]. Otherwise, the candidate region was considered as a false positive.

The free-response receiver operating characteristic (FROC) and ROC curves are used for evaluation of the overall performance of the CAD systems for various operating points. The FROC curve shows the relationship between the sensitivity and the number of false positives, which can be obtained by thresholding a certain parameter of the CAD system or the output of the classifier. Figure 10 shows the FROC curve for the overall performance of a scheme from Arimura et al. [26] for a database by use of LDA with the leave-one-out-by-patient test method. The SBDI is the shape-based difference image. From Figure 10, we can evaluate that the CAD system with the SBDI features is better than that without the features. 
It is very important for some CAD systems to evaluate not only the sensitivity of lesions, but also the accuracy of lesion segmentation. For example, MS lesions present temporal changes in the shape, location, and area between patients [3-6]. Therefore, neuroradiologists need to know the differences in the shape, location, and area between two studies, which should be provided by the CAD systems for the MS. For such CAD systems, the segmentation accuracy should be evaluated by using some indicators such as overlap measure [63], which means the degree of coincidence between the candidate region obtained by a method and the gold standard region by a manual method, as follows:

Overlap measure $(\%)=\frac{n(T \cap C)}{n(T \cup C)} \times 100$

where $T$ was the gold standard region manually determined by radiologists, $C$ was the region automatically determined by using a method, $n(T \cup C)$ was the number of logical OR pixels between $T$ and $C$, and $n(T \cap C)$ was the number of logical AND pixels between $T$ and $C$. In addition, the following similarity index is also used for evaluation of segmentation accuracy:

Similarity index $=\frac{2 n(T \cap C)}{n(T)+n(C)}$

where $n(T)$ was the number of the gold standard region and $n(C)$ was the number of the candidate region.

Moreover, it is essential for us to assess the radiologist's performance as well as the CAD performance itself. By use of the ROC curve based on an observer test, we can quantitatively estimate the potential of the CAD system that can improve the radiologist's performance, although the observer test is performed in the laboratory. The effects of the CAD systems on the radiologist's performance have been investigated by using the ROC analysis, which was described in Refs. 23,24,63.

Figure 10. FROC curve for the overall performance of a scheme from Arimura et al. [26] for a database by use of LDA with the leave-one-out-by-patient test method. The SBDI is the shape-based difference image.

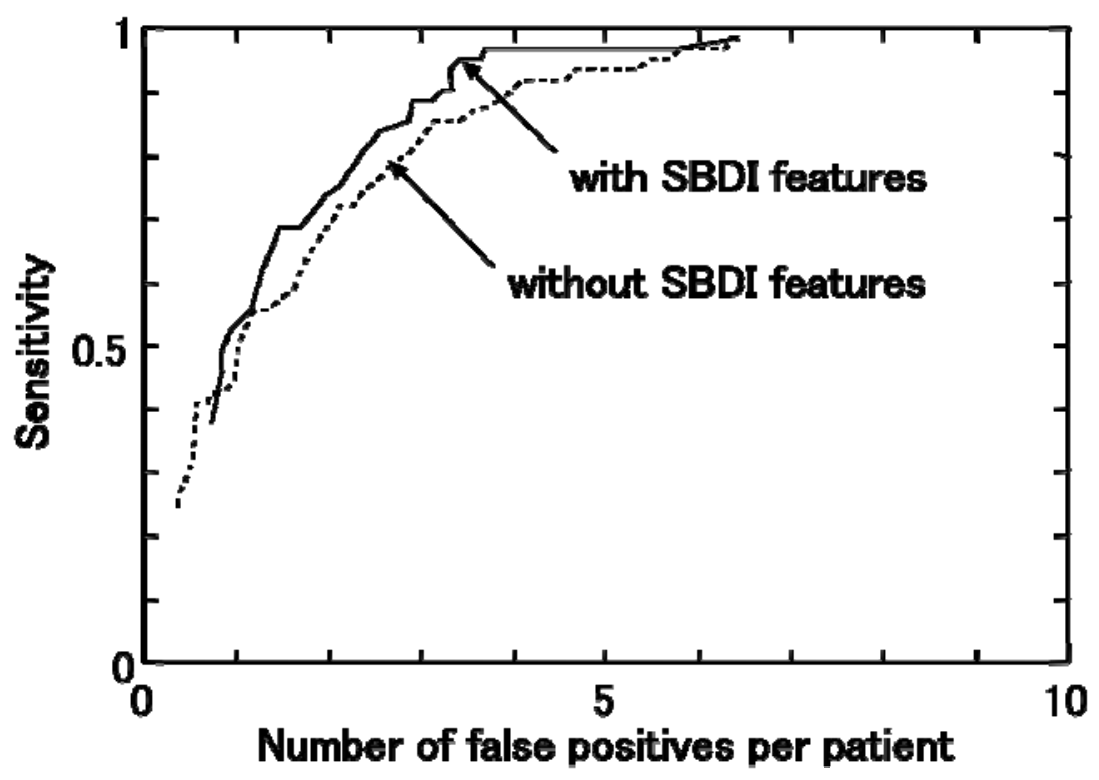




\section{Application examples of CAD for brain diseases}

\subsection{Intracranial aneurysm}

One of the purposes of the medical checks for brain is to detect asymptomatic unruptured aneurysms using the MR angiography (MRA) [1]. Ruptures of intracranial aneurysms cause subarachnoid hemorrhage (SAH), which kills more than 10,000 persons each year in North America [64]. People who have a family history of aneurysms or who have polycystic kidneys, coarctation of the aorta, or collagen vascular disease, could be put at an increased risk for aneurysms. In those people, it is very important to detect unruptured aneurysms as early as possible and to treat or follow up the aneurysms. However, it is difficult and time consuming for radiologists to detect small aneurysms, because of their overlap with adjacent vessels or unusual locations in maximum intensity projection (MIP) images of MRA. Therefore, a number of CAD systems have been developed for assisting radiologists in detection of intracranial aneurysms [25,26,41,65,66]. Table 1 shows a comparison of methods and results of CAD systems for detection of intracranial aneurysms using MRA. Kobashi et al. [66] proposed a CAD system for detecting intracranial aneurysms in MRA images based on estimation of the fuzzy degrees for each aneurysm candidate, which denoted whether a candidate is an aneurysm. Hayashi et al. [41] developed a curvature-based display system that shows volume-rendered images with overlaid curvature indices determined from MRA images. However, neuroradiologists should identify aneurysm candidates by using color-coded curvatures corresponding to the degree of aneurysm, because the system did not automatically detect intracranial aneurysms. Uchiyama et al. [65] developed an automated detection system for aneurysms based on a gradient concentrate filter, which was applied to segmented vessels for enhancement of aneurysm. Arimura et al. developed a CAD system for detection of intracranial aneurysms using MRA images [25,26]. The selective enhancement filters for dots [40], which can enhance dot-like objects, were applied to isotropic 3-D MRA images. Useful image features related to small protrusions were extracted from the shape-based difference (SBD) regions obtained by the SBD image technique. A protrusion of a SBD region could be a small aneurysm, but a thin or very small region of a SBD region could be a false positive. Figure 11 shows the illustrations of images produced in each step of the SBD image technique. We tested the computerized scheme on 53 cases with 61 aneurysms and 62 non-aneurysm cases based on a leave-one-out-by-patient test method. As a result, the CAD system achieved a high sensitivity of $97 \%$ with 3.8 false positives per patient by use of the SBDI technique. 
Table 1. Comparison of methods and performance of CAD systems for detection of intracranial aneurysms using MRA.

\begin{tabular}{cccccc}
\hline Authors & $\begin{array}{c}\text { Lesion } \\
\text { enhancement }\end{array}$ & Classifier & $\begin{array}{c}\text { No.cases } \\
\text { (No.aneurysms) }\end{array}$ & $\begin{array}{c}\text { Sensitivity } \\
(\%)\end{array}$ & $\begin{array}{c}\text { No.FPs per } \\
\text { case }\end{array}$ \\
\hline Kobashi et al. [66] & Fuzzy degree & Fuzzy & $16(19)$ & 100 & 6.4 \\
Hayashi et al. [41] & $\begin{array}{c}\text { Curvature } \\
\text { Gradient } \\
\text { concentrate } \\
\text { filter }\end{array}$ & - & $18(23)$ & - & 1.9 \\
Achiyama et al. [65] & QDA & $7(7)$ & 100 & 3.8 \\
\hline
\end{tabular}

*QDA: quadratic discriminant analysis

**LDA: linear discriminant analysis

Figure 11. Illustrations of images produced in each step of the shape-based difference image technique: (a) segmented vessel, (b) distance transformation, (c) determination of vessel skeleton, (d) logical AND between skeleton image and distance transformed image, (e) smoothing skeleton image with distance value by rind filter, and (f) vessel with suppressed local change on thickness [26].

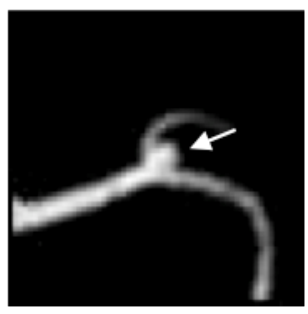

(a)

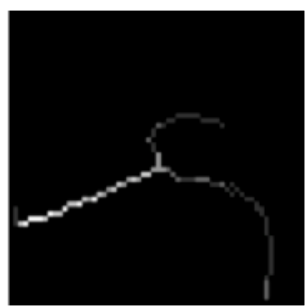

(d)

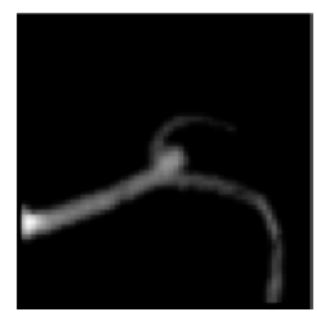

(b)

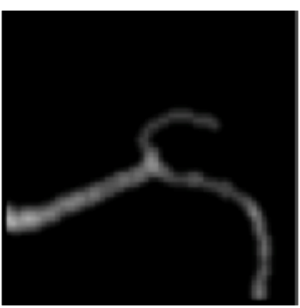

(e)

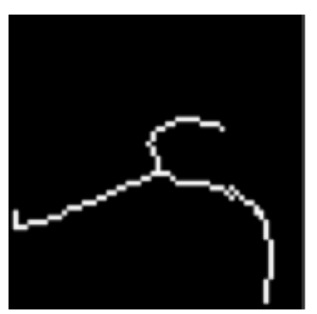

(c)

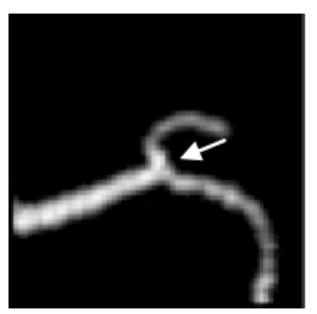

(f)

\subsection{White matter hyperintensities in vascular dementia}

Subcortical vascular dementia $(\mathrm{VaD})$ is a neurodegenerative vascular disorder that leads to a progressive decline in memory and cognitive functioning, and is considered the second most common cause of dementia in Japan. The prevalence of the $\mathrm{VaD}$ in people older than 70 years in Japan is around 27\% including mix dementia of Alzheimer's disease and $\mathrm{VaD}$ [67]. Ischemic lesions in $\mathrm{VaD}$ 
patients show hyperintense regions in the cerebral white matter, i.e., periventricular hyperintense $(\mathrm{PVH})$ regions and white matter hyperintense $(\mathrm{WMH})$ regions, on FLAIR images or T2-weighted images at MR imaging. It has been reported that the degree of the symptom of the $\mathrm{VaD}$ correlated with an area ratio of WMH region to the whole brain region (WMH region ratio) [68-71], which could provide diagnostic information for the treatment of $\mathrm{VaD}$. Thus, it is very important to estimate the $\mathrm{WMH}$ region ratio for evaluating the degree of the $\mathrm{VaD}$. Although neuroradiologists attempt to manually evaluate the WMH regions, it is very laborious and demanding in routine clinical practice. Therefore, investigators have reported CAD systems for detection of WMH including lacunar infarct using MR images [28, 72-76]. Mohamed et al. [72] developed a segmentation method for white-matter lesions including $\mathrm{VaD}$, multiple sclerosis, and lacunar infarcts based on a modified k-mean-neighbor method using three-dimensional (3D) MR images. Anbeek et al. [73] presented a method for automated segmentation of WMHs in MR images by using a k-nearest neighbor method, which builds a feature space from voxel intensity features and spatial information. Admiraal-Behloul et al. [74] proposed a automatic segmentation method of WMHs based on a fuzzy C-mean algorithm using three different MR images: proton density, T2-weighted, and FLAIR images. Uchiyama et al. [75] developed a computerized scheme for detection of lacunar infarct by using a rule-based method and an SVM. Their method detected $96.8 \%$ of lacunar infarct with 0.76 false positives. Zhiqiang et al. [76] suggested an approach for segmentation of white-matter lesions related to cerebrovascular disease based on a SVM on a 3D MR image. On the other hand, for assisting neuroradiologists in the diagnosis of $\mathrm{VaD}$, Yamashita et al. [28] developed an automated method for detecting and segmenting WMH regions based on conventional MR images (T1-weighted, T2-weighted, and FLAIR images). Their method attained a sensitivity of $90 \%$ with 4.0 false-positives per slice in detection of WMH regions. Table 2 shows a comparison of methods and performance of CAD systems for segmentation of WMH using MR images. This table does not include the results of Mohamed et al., Uchiyama et al., and Zhiqiang et al., because they did not explicitly state the similarity index in their papers. According to Table 2, the sensitivities and similarity indices were higher than $90 \%$ and $74 \%$, respectively. The researchers of CAD systems for detection of WMHs focus on segmentation accuracy, because the neuroradiologists should measure the WMH areas in MR images for diagnosis and follow-up of patients. In a method of Yamashita et al. [28], many false-positives were removed based on the outputs of four ANNs, which were considered the possibility of the WMH regions. Figure 12 shows the illustrations of (a) an original T1-weighted image, (b) an original FLAIR image with hyperintense regions, (c) a FLAIR-T1 subtraction image. As shown in Figure 12, a majority of WMH regions are in the periventricular region, which was divided into the anterior horn, posterior horn, and the periventricular body [77]. The left and right horns were located about $45^{\circ}, 135^{\circ}, 225^{\circ}$, and $315^{\circ}$ from the centroid of a brain. Therefore, each brain was divided into four quarters $\left(90^{\circ}\right)$ based on this clinical evidence. Four ANNs were constructed for four divided brain regions. Input features for the ANN were mean, maximum, and minimum pixel values of a candidate in T1-weighted, T2-weighted, FLAIR images, and the FLAIR-T1 subtraction images, effective diameter, circularity, and the distance between a candidate and the centroid of a brain region. 
Table 2. Comparison of methods and performance of CAD systems for detection of white matter hyperintensities (WMHs) in MR images.

\begin{tabular}{|c|c|c|c|c|}
\hline Authors & $\begin{array}{c}\text { Lesion } \\
\text { enhancement }\end{array}$ & Classifier & $\begin{array}{c}\text { No.cases } \\
\text { (No.WMHs) }\end{array}$ & SI* \\
\hline Anbeek et al. [75] & $\begin{array}{l}\text { k-nearest } \\
\text { neighbor }\end{array}$ & $\begin{array}{l}\text { k-nearest } \\
\text { neighbor }\end{array}$ & $19(-)$ & 0.81 \\
\hline $\begin{array}{l}\text { Admiraal-Behloul et al. } \\
\qquad[74]\end{array}$ & $\begin{array}{c}\text { Fuzzy C-mean } \\
\text { algorithm }\end{array}$ & Fuzzy & $100(100)$ & 0.75 \\
\hline Yamashita et al. [28] & $\begin{array}{c}\text { FLAIR**-T1 } \\
\text { difference } \\
\text { image }\end{array}$ & $\mathrm{ANN}^{* * *}$ & $9(85)$ & 0.74 \\
\hline
\end{tabular}

*SI: similarity index

**FLAIR: fluid-attenuated inversion-recovery

***ANN: artificial neural network

Figure 12. Illustrations of (a) an original T1-weighted image, (b) an original fluidattenuated inversion-recovery (FLAIR) image with hyperintense regions, (c) a subtraction image between the FLAIR image and T1-weighted image [28].

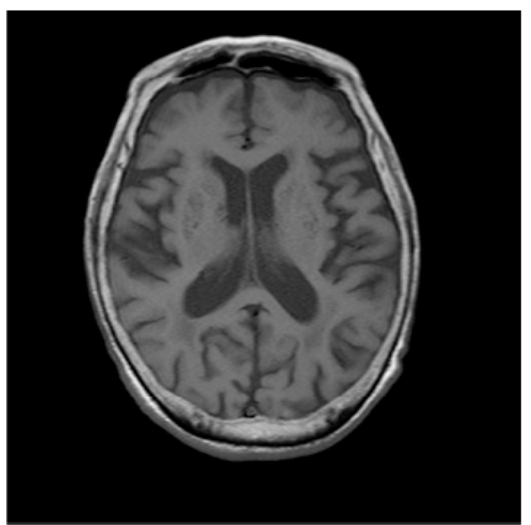

(a)

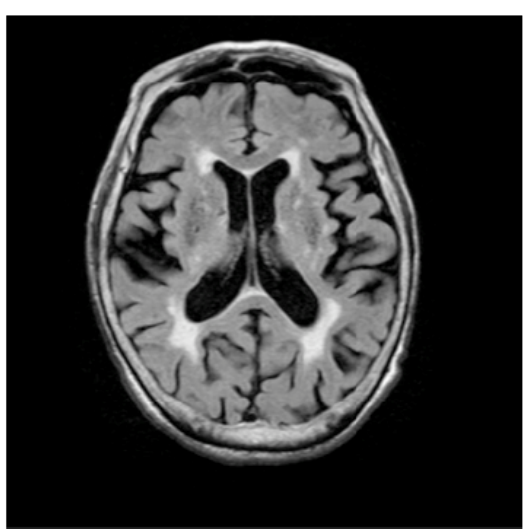

(b)

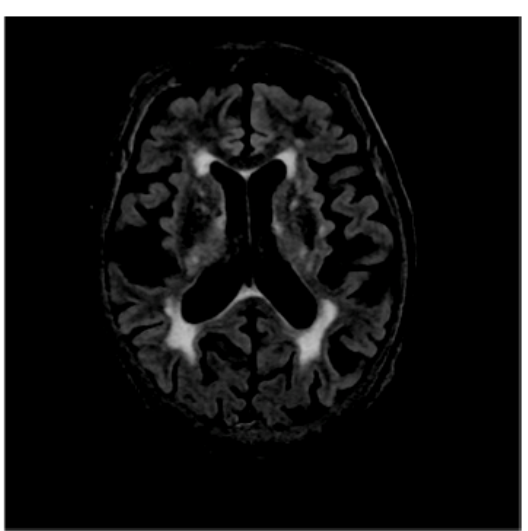

(c)

\subsection{Alzheimer's disease}

Alzheimer's disease (AD) is an irreversible, progressive dementing disorder, which slowly deteriorates memory and thinking skills [78]. Since the incidence of the AD is strongly linked to the age, the $\mathrm{AD}$ is one of the major public health problems in countries with the longer life expectancy. According to recent estimates, as many as 2.4 million to 4.5 million Americans and 1.8 million Japanese have $\mathrm{AD}[78,79]$. AD is associated with the atrophy of gray matter in the cerebral cortex, which leads to a volume decrease of the cerebral cortex or a volume increase of cerebrospinal fluid (CSF) in cerebral sulci and lateral ventricles (LVs), which can be measured in magnetic resonance 
(MR) images. In order to provide appropriate care for $\mathrm{AD}$ patients, it is very important to quantitatively evaluate the degree of the atrophy in the cerebral cortex in the early stages of AD. Neuroradiologists attempt to estimate the degree of atrophy by capturing atrophic image features on MR images, but it is very difficult and time consuming in routine clinical practice. Therefore, a number of automated methods have been studied for identification of AD patients among the large number of patients with dementia [27,80-83]. In recent years, a whole-brain unbiased objective technique, known as voxel-based morphometry (VBM) [84], has been developed for characterizing differences in the local composition of brain tissue using MR images, and can objectively map gray matter loss on a voxel-by-voxel basis. Hirata et al. [80] developed a software based on the voxel-based specific region analysis for Alzheimer's disease (VSRAD), which can automatically analyze 3D T1weighted MRI data as a series of segmentation, anatomical standardization and smoothing using a VBM software (statistical parametric mapping: SPM) and Z-score analysis. The averaged value of positive Z-scores with respect to the volumes in the specific region of interest in a Z-score map was as the threshold for classifying $\mathrm{AD}$ cases and non-AD cases. Li et al. [81] employed a SVM for characterization of the hippocampal volume changes in $\mathrm{AD}$ and differentiation of $\mathrm{AD}$ patients from healthy control subjects. Klöppel et al. [82] also used linear support vector machines to classify the gray matter segment of T1-weighted MR scans from AD patients and normal elderly individuals. In their method, MR images were segmented into gray matter (GM), white matter and CSF using SPM. Colliot et al. [83] developed an automated segmentation method for hippocampal volumetry to help distinguish between patients with $\mathrm{AD}$, patients with mild cognitive impairment (MCI), and elderly controls, by using established criteria for patients with AD and MCI as the reference standard. Table 3 shows the comparison of methods and performances of CAD systems for Alzheimer's disease using MR images. The accuracy for classification of $\mathrm{AD}$ patients ranges from $82.7 \%$ to $89.6 \%$, which seems to be relatively high accuracy.

We attempt to develop a CAD system for identification of patients with cerebral atrophy due to Alzheimer's disease based on a pattern recognition technique using 3-D MR images. Our CAD system consists of determination of atrophic image features and identification of AD patients. First, brain, white matter and gray matter regions were determined based on a level set method. Second, CSF regions in cerebral sulci and LVs were extracted by wrapping the brain with a level set function. Third, the cerebral cortical thickness was measured based on normal vectors as a distance from the white matter surface to the brain surface. To select mean cortical thicknesses in the most effective subregions for distinguishing $\mathrm{AD}$ patients from non-AD patients, we employed a Golub statistic [85,86], which can be interpreted as the degree of separation between the two classes. Finally, AD cases were classified from non-AD cases based on a support vector machine (SVM) classifier, which learned atrophic image features, i.e., white matter and gray matter volumes, CSF volume, and cerebral cortical

thickness. The SVM classifier was constructed with a Gaussian kernel exp $\left(-\gamma\|\mathrm{x}-\mathrm{y}\|^{2}\right)$ by using an open source software SVM light [87]. 
Table 3. Comparison of methods and performance of CAD systems for Alzheimer's disease using MR images.

\begin{tabular}{|c|c|c|c|c|c|}
\hline Authors & Classifier & Feature & $\begin{array}{c}\text { No.cases } \\
\text { (No.AD cases) }\end{array}$ & Accuracy (\%) & AUC* \\
\hline Hirata et al. [80] & Thresholding & Z-score** & $72(31)$ & 87.8 & 0.949 \\
\hline Li et al. [81] & $\mathrm{SVM}^{* * *}$ & $\begin{array}{c}\text { Hippocampal } \\
\text { volume }\end{array}$ & $39(19)$ & 89.6 & - \\
\hline Kloppel et al. [82] & SVM & $\begin{array}{l}\text { Gray matter } \\
\text { voxels, etc. }\end{array}$ & $158(67)$ & 89.0 & - \\
\hline Colliot et al. [83] & Thresholding & $\begin{array}{c}\text { Hippocampal } \\
\text { volume }\end{array}$ & $50(25)$ & 84.0 & - \\
\hline Arimura et al. [27] & SVM & $\begin{array}{c}\text { Cortical } \\
\text { thickness, etc. }\end{array}$ & $54(29)$ & 82.7 & 0.909 \\
\hline
\end{tabular}

*AUC : area uder receiver-operating characteristic curve

$* *$ Z-score $=([$ control mean $]-[$ individual value $]) /(\operatorname{controlSD})$

$* * *$ SVM : support vector machine

Figure 13 shows the segmentations of brain regions (outer white line) and white matter regions (inner white line) obtained by the proposed method from a non-AD patient in multiple coronal planes. As a result, the area under a ROC curve (Az value) obtained by the CAD system was 0.909 based on a leave-one-out test in identification of AD patients among 54 cases. The preliminary results showed that the proposed method may be promising for identification of AD patients.

Figure 13. Segmentations of brain regions (outer white line) and white matter regions (inner white line) obtained by the proposed method from a non-AD patient in multiple coronal planes [27].
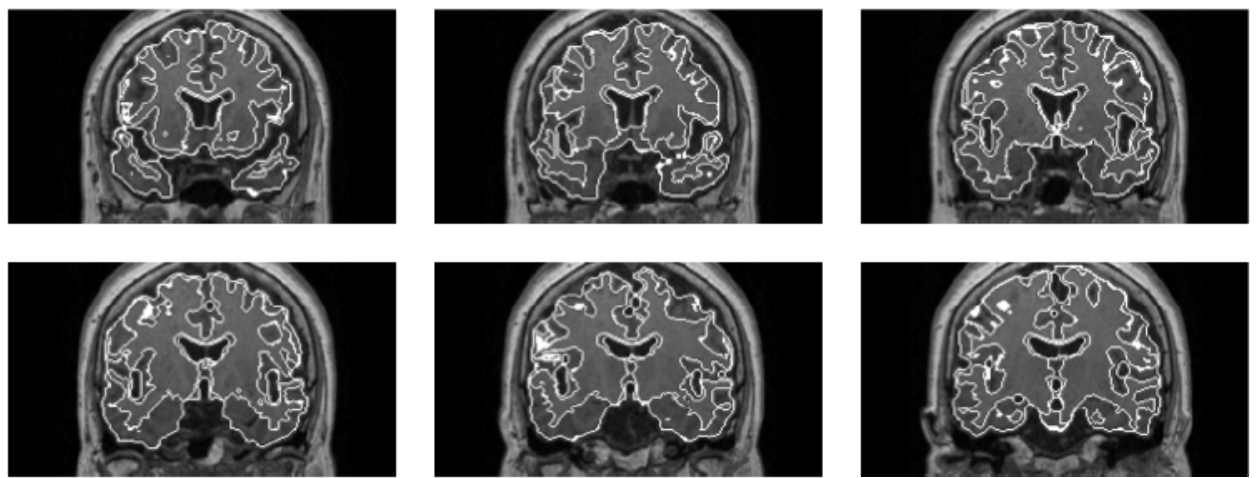

\subsection{Multiple sclerosis}

Multiple sclerosis (MS) is a progressive neurological disorder, which is caused by structural damages of axons and their myelin sheathes in the central nervous system. MS lesions present temporal changes in shape, location, and area between patients, and thus it is necessary for radiologists to accurately detect and evaluate MS lesions [3-6]. However, the accurate assessment of each lesion in 
magnetic resonance (MR) images would be a demanding and time-consuming task, and also a manual measurement could be subjective and have poor reproducibility. Therefore, a number of semiautomated or automated methods have been proposed for identifying and/or segmenting MS lesions based on two or three-dimensional (3-D) MR images. Alfano et al. [88] proposed an automated approach based on relaxometric and geometric features for classification of MS lesions in 1.5 Tesla 3D MR images. Boudraa et al. [89] applied the FCM algorithm to 1.5 Tesla two-dimensional (2-D) MR images for classifying normal and abnormal brain structures. Leemput et al. [90] proposed an automated method by using an intensity-based tissue classification and a stochastic model for detection of MS lesions in 1.5 Tesla 3-D images. Zijdenbos et al. [91] developed an automated framework for the pipeline analysis of MS lesions in MRI data. Khayati et al. [92,93] developed an automated method for segmentation of MS lesions in brain MR FLAIR images using an adaptive mixture method and a markov random field model in 1.5 Tesla 3-D MR images. Their proposed approach was based on a Bayesian classifier to obtain and upgrade the class conditional probability density function and a prior probability of each class. Table 4 shows the comparison of methods and results of CAD systems for detection of MS in MR images. The researchers for MS CAD systems are not likely to present the sensitivity and the number of false positives except for our group. Instead, they reported the similarity indices, which range from 0.51 to 0.75 .

Table 4. Comparison of methods and performance of CAD systems for detection of MS in MR images.

\begin{tabular}{cccc}
\hline Authors & Segmentation & $\begin{array}{c}\text { No.cases (No.MS } \\
\text { lesions) }\end{array}$ & SI* \\
\hline Alfano et al. [88] & T1, T2, proton density & $84(-)$ & - \\
Boudraa et al. [89] & maps & $10(-)$ & 0.62 \\
Leemput et al. [90] & Suzzy C-Means & $50(-)$ & 0.51 \\
Zijdenbos et al. [91] & Pipeline analysis & $29(-)$ & 0.68 \\
Khayati et al. $[92,93]$ & AMM**, MRF*** & $20(-)$ & 0.75 \\
Yamamoto et al. $[30,31]$ & Region growing, LSM & $3(168)$ & 0.77 \\
\hline
\end{tabular}

*SI: similarity index

**AMM: adaptive mixtures method

***MRF: Markov random field model

+LSM: level set method

We are developing a CAD system for detection of MS lesions in brain 3.0 Tesla two-dimensional MR images based on a level set method [30, 31]. In the proposed method, the level set method was employed for reduction of false positives as well as accurate segmentation of MS lesions. A number of false positive candidate regions were removed according to the output of an SVM, which was learned with image features and tested based on a leave-one-candidate-out test method. We applied the proposed method to 49 slices selected from 6 studies of three MS cases including $168 \mathrm{MS}$ lesions. As a result, the sensitivity for detection of MS lesions was $81.5 \%$ with 2.9 false positives per slice based on 
a leave-one-candidate-out test, and the degree of coincidence between MS lesions detected by the proposed method and two neuroradiologists was 0.768 on average. These results indicate the proposed method would be useful for assisting neuroradiologists in assessing MS in clinical practice.

\subsection{Brain glioma}

Accurate noninvasive radiologic diagnosis of brain tumors is desirable for appropriate treatment planning. The diagnosis of brain tumors is usually made subjectively using MR imaging, and its accuracy may be limited by the presence of atypical cases or by a radiologist's insufficient clinical experience. A computerized method that is capable of providing objective information about an image may assist radiologists in the classification of brain tumors. ANNs have been reported to improve the diagnostic performance of radiologists in several fields [94-102]. A few groups applied ANNs including a self-organizing maps (SOMs) to classify intracranial diseases including brain tumors, pituitary adenoma, craniopharyngioma, and Rathke's cleft cyst. Vijayakumar et al. [103] designed a computer-assisted method to perform segmentation of brain tumor on apparent diffusion coefficient (ADC) images and evaluate its grade (malignancy state), using a mixture of unsupervised ANN and hierarchical multiresolution wavelet. As a result, the average sensitivity and the specificity for high or low grade tumor, edema, necrosis, CSF and normal tissue are observed to be 0.86 and 0.93 , respectively. Kitajima et al. [104] applied an ANN for differential diagnosis among pituitary adenoma, craniopharyngioma, and Rathke's cleft cyst with MR images and retrospectively evaluate the effect of ANN output on radiologists' performance. The ANN showed high performance in differentiation among the three lesions (AUC $=0.990)$. The average AUC for all radiologists for differentiation among the three lesions increased significantly from 0.910 to $0.985(\mathrm{P}=0.0024)$ when they used the computer output. We also constructed an ANN for the differential diagnosis of intra-axial cerebral tumors on MR images and evaluated the effect of ANN outputs on radiologists' diagnostic performance [60]. The radiologists collected MR images of 126 patients with intra-axial cerebral tumors (58 high-grade gliomas, 37 low-grade gliomas, 19 metastatic tumors, and 12 malignant lymphomas). We constructed a single 3-layer feed-forward ANN, as shown in Figure 9, with a Levenberg-Marquardt algorithm. The ANN was designed to differentiate among 4 categories of tumors (high-grade gliomas, low-grade gliomas, metastases, and malignant lymphomas) with use of 2 clinical parameters and 13 findings in MR images. Subjective ratings for the 13 findings were provided independently by 2 attending radiologists. Originally, the rating scores were integer, nominal values depending on grade or location, but all inputs used in this study for the ANN were linearly normalized from -0.9 to 0.9 . All 126 cases were used for training and testing of the ANN based on a leave-one-out-by-case method.

In the observer test, MR images were viewed by 9 radiologists, first without and then with ANN outputs. Each radiologist's performance was evaluated through a ROC analysis on a continuous rating scale. The averaged area under the ROC curve for ANN alone was 0.949. The diagnostic performance of the 9 radiologists increased from 0.899 to $0.946(\mathrm{P}<0.001)$ when they used ANN outputs. The ANN can provide useful outputs as a second opinion to improve radiologists' performance in the differential diagnosis of intra-axial cerebral tumors seen on MR imaging. 


\section{Conclusions}

This review paper describes CAD systems from the basics to several applications of the CAD for brain diseases in neuroradiology. However, a number of researchers including our group have just begun researching various kinds of CAD systems for brain diseases. Neuroradiologists expect that the CAD systems can assist them in diagnosing brain diseases by providing useful information. CAD researchers attempt to develop very useful CAD systems, which can improve neuroradiologist's abilities to diagnose patients based on a synergistic effect between the computer and neruroradiologists. Many advanced countries with a long life expectancy such as Japan and the United States are moving toward an aging society, and thus the number of patients with brain diseases including some dementing disorders will increase with the rise in average life expectancy. Therefore, the CAD systems for brain diseases will become more essential for neuroradiologists in clinical practice in the near future.

\section{Acknowledgments}

We are grateful to Kenji Suzuki, The University of Chicago, who gave us a chance to submit this review paper. We thank Takashi Yoshiura, Kyushu University; Yukunori Korogi, and Shingo Kakeda, University of Occupational and Environmental Health; Kazuhiro Tsuchiya, Kyorin University; Yoshiharu Higashida, Fukai Toyofuku, Masafumi Ohki, Junji Morishita, Seiji Kumazawa, and Yasuo Kawata, Kyushu University, for many helpful suggestions and comments on the researches presented in this review paper, and Robert Chartrand for improving the manuscript. This work was partially supported by the Ministry of Education, Science, Sports and Culture, Japan, Grant-in-Aid for Scientific Research (C), 19591423, 2007.

\section{References}

1. Guideline for Brain Dock; Japanese Society for Detection of Asymptomatic Drain Diseases, 2008, (in Japanese).

2. The World Health Report 2006. Working Together for Health.; World Health Organization, 2006, (available online: http://www.who.int/whr/2006/en/index.html).

3. Filippi, M.; Campi, A.; Martinelli, V.; Colombo, B.; Scotti, G.; Comi, G. Brain and spinal cord MR in benign multiple sclerosis: a follow-up study. Journal of the Neurological Sciences 1996, 143, 143-149.

4. Guttmann, C.R.; Kikinis, R.; Anderson, M.C.; Jakab, M.; Warfield, S.K.; Killiany, R.J.; Weiner, H.L.; Jolesz, F.A. Quantitative follow-up of patients with multiple sclerosis using MRI: resproducibility. Journal of Magnetic Resonannce Imaging 1999, 9, 509-518.

5. Weiner, H.L; Guttmann, C.R; Khoury, S.J; Orav, E.J; Hohol, M.J; Kikinis, R; Jolesz, F.A. Serial magnetic resonance imaging in multiple sclerosis: correlation with attacks, disability, and disease stage. Journal of Neuroimmunology 2000, 104, 164-173. 
6. Goldberg-Zimring D.; Achiron, A.; Guttmann, C.R.G.; Azhari, H. Three-Dimensional analysis of the geometry of individual multiple sclerosis lesions: Detection of shape changes over time using spherical harmonics. Journal of Magnetic Resonannce Imaging 2003, 18, 291-301.

7. Hoffmann, K.R.; Doi, K.; Chan, H.P.; Fencil, L.; Fujita, H.; Muraki, A. Automated tracking of the vascular tree in DSA images using a double-square-box region-of-search algorithm. Proc SPIE 1986, 626, 326-333.

8. Chan, H-P.; Doi, K.; Galhotra, S.; Vyborny C.J.; MacMahon, H.; Jokich, P.M. Image feature analysis and computer-aided diagnosis in digital radiography.1. Automated detection of microcalcifications in mammography. Medical Physics 1987, 14, 538-548.

9. Giger, M.L.; Doi, K.; MacMahon, H. Image feature analysis and computer aided diagnosis in digital radiography. 3. Automated detection of nodules in peripheral lung fields. Medical Physics 1988, 15, 158-166.

10. Chan, H.-P.; Doi, K.; Vyborny, C.J; Schmidt, R.A.; Metz, C.E.; Lam, K.L.; Ogura, T.; Wu, Y.; MacMahon, H.; Sickles, E.A. Improvement in radiologists' detection of clustered microcalcifications on mammograms - The potential of computer-aided diagnosis. Investigative Radiology 1990, 25, 1102-1110.

11. Huo, Z.; Giger, M.L.; Vyborny, C.J.; Metz, C.E. Effectiveness of CAD in the diagnosis of breast cancer: An observer study on an independent database of mammograms. Radiology 2002, 224, $560-568$.

12. Wu, Y.T.; Wei, J.; Hadjiiski, L.M.; Sahiner, B.; Zhou, C.; Ge, J.; Shi, J.; Zhang, Y.; Chan, H.-P. Bilateral analysis based false positive reduction for computer-aided mass detection. Medical Physics 2007, 34, 3334-3344.

13. Chan, H-P.; Wei, J.; Zhang, Y.; Helvie, M.A.; Moore, R.H.; Sahiner, B.; Hadjiiski, L.: Kopans, D.B. Computer-aided detection of masses in digital tomosynthesis mammography: comparison of three approaches. Medical Physics 2008, 35, 4087-4095.

14. Arimura, H.; Katsuragawa, S.; Suzuki, K.; Li, F.; Shiraishi, J.; Doi, K. Computerized scheme for automated detection of lung nodules in lowdose CT images for lung cancer screening. Academic Radiology 2004, 11, 617-629.

15. Uchiyama, Y.; Katsuragawa, S.; Abe, H.; Shiraishi, J.; Li, F.; Li, Q.; Zhang, C.-T.; Suzuki, K.; Doi, K.; Quantitative computerized analysis of diffuse lung disease in high-resolution computed tomography. Medical Physics 2003, 30, 2440-2464.

16. Li, F.; Arimura, H.; Suzuki, K.; Shiraishi, J.; Li, Q.; Abe, H.; Engelmann, R.; Sone, S.; MacMahon, H.; Doi, K. Computer-aided detection of peripheral lung cancers missed at CT: ROC analyses without and with localization. Radiology 2005, 237, 684-690.

17. Suzuki, K.; Li, F.; Sone, S.; Doi, K. Computer-aided diagnostic scheme for distinction between benign and malignant nodules in thoracic low-dose CT by use of massive training artificial neural network. IEEE Transactions on Medical Imaging 2005, 24, 1138-1150.

18. Li, Q.; Li, F.; Doi, K. Computerized detection of lung nodules in thin-section CT images by use of selective enhancement filters and an automated rule-based classifier. Academic Radiology 2008, 15, 165-175.

19. Yoshida, H.; Masutani, Y.; MacEneaney, P.; Rubin, D.; Dachman, A.H. Computerized detection of polyps in CT colonography. Radiology 2002, 222, 327-336. 
20. Nappi, J.; Yoshida, H. Feature-guided analysis for reduction of false positives in CAD of polyps for CT colonography. Medical Physics 2003, 30, 1592-1601.

21. Suzuki, K.; Yoshida, H.; Näppi, J.; Dachman, A.H. Massive-training artificial neural network (MTANN) for reduction of false positives in computer-aided detection of polyps: Suppression of rectal tubes. Medical Physics 2006, 33, 3814-3824.

22. Suzuki, K.; Yoshida, H.; Näppi, J.; Armato, S.G. 3rd; Dachman, A.H. Mixture of expert 3D massive-training ANNs for reduction of multiple types of false positives in CAD for detection of polyps in CT colonography. Medical Physics 2008, 35, 694-703.

23. Hirai, T.; Korogi, Y.; Arimura, H.; Katsuragawa, S.; Kitajima, M.; Yamura, M.; Yamashita, Y.; Doi, K. Intracranial aneurysms at MR angiography. Effect of computer-aided diagnosis on radiologists' detection performance. Radiology 2005, 237, 605-610.

24. Kakeda, S.; Korogi, Y.; Arimura, H.; Hirai, T.; Katsuragawa, S.; Aoki, T.; Doi, K. Diagnostic accuracy and reading time to detect intracranial aneurysms on MR angiography using a computeraided diagnosis system. American Journal of Roentgenology 2008, 190, 459-465.

25. Arimura, H.; Li, Q.; Korogi, Y.; Hirai, T.; Abe, H.; Yamashita, Y.; Katsuragawa, S.; Ikeda, R.; Doi, K. Automated computerized scheme for detection of unruptured intracranial aneurysms in three-dimensional MRA. Academic Radiology 2004, 11, 1093-1104.

26. Arimura, H.; Li, Q.; Korogi, Y.; Hirai, T.; Katsuragawa, S.; Yamashita, Y.; Tsuchiya, K.; Doi, K. Computerized detection of intracranial aneurysms for 3D MR angiography: Feature extraction of small protrusions based on a shape-based difference image technique. Medical Physics 2006, 33, 394-401.

27. Arimura, H.; Yoshiura, T.; Kumazawa, S.; Tanaka, K.; Koga, H.; Mihara, F.; Honda, H.; Sakai, S.; Toyofuku, F.; Higashida, Y. Automated method for identification of patients with Alzheimer's disease based on three-dimensional MR images. Academic Radiology 2008, 15, 274-284.

28. Yamashita, Y.; Arimura, H.; Tsuchiya, K. Computer-aided detection of ischemic lesions related to subcortical vascular dementia on magnetic resonance images. Academic Radiology 2008, 15, 978985.

29. Fujita, H.; Uchiyama, Y.; Nakagawa, T.; Fukuoka, D.; Hatanaka, Y.; Hara, T.; Lee, G.N.; Hayashi, Y.; Ikedo, Y.; Gao, X.; Zhou, X. Computer-aided diagnosis: The emerging of three CAD systems induced by Japanese health care needs. Computer Methods and Programs in Biomedicine 2008, 92, 238-248.

30. Yamamoto, D.; Arimura, H.; Kakeda, S.; Magome, T.; Yamashita, Y.; Ohki, M.; Toyofuku, F.; Higashida, Y.; Korogi, Y. Computer-aided detection of multiple sclerosis lesions based on three types of blain MR images. International Journal of Computer Assisted Radiology and Surgery (CARS) 2008, 3, 202-203.

31. Yamamoto, D.; Arimura, H.; Kakeda, S.; Magome, T.; Yamashita, Y.; Ohki, M.; Toyofuku, F.; Higashida, Y.; Korogi, Y. Computerized detection of multiple sclerosis lesions based on 3.0T two-dimensional magnetic resonance images. The Institute of Electronics, Information and Communication Engineers (IEICE) Technical Report 2009, MI2008-171, 505-506.

32. Duda, R.O.; Hart, P.E.; Stork, D.G. Pattern classification, 2nd ed.; Wiley-Interscience: New York, NY, USA, 2000. 
33. Nagao, M.; Matsuyama, T. Edge preserving smoothing. Computer Graphics and Image Processing 1979, 9, 394-407.

34. Lee, Y.; Takahashi, N.; Tsai, D. Adaptive partial median filter for early CT signs of acute cerebral infarction. International Journal of Computer Assisted Radiology and Surgery 2007, 2, 105-115.

35. Talairach, J.; Tournoux, P. Co-planar stereotaxic atlas of the human brain: 3-dimensional proportional system-an approach to cerebral imaging, Thieme Medical Publishers: New York, NY, USA, 1988.

36. Giger, M.L.; Doi, K.; MacMahon, H. Image feature analysis and computer-aided diagnosis in digital radiography. 3. Automated detection of nodules in peripheral lung fields. Medical Physics 1988, 15, 158-166.

37. Yamamoto, S.; Matsumoto, M.; Tateno, Y.; Iinuma, T.; Matsumoto, T. Quoit Filter - A new filter based on mathematical morphology to extract the isolated shadow, and its application to automatic detection of lung cancer in x-ray CT. International Conference on Pattern Recognition (ICPR) 1996, 2, 3.

38. Sato, Y.; Nakajima, S.; Shiraga, N.; Atsumi, H.; Yoshida, S.; Koller, T.; Gerig, G.; Kikinis, R. Three-dimensional multi-scale line filter for segmentation and visualization of curvilinear structures in medical images. Medical Image Analysis 1998, 2, 143-168.

39. Sato, Y.; Westin, C.-F.; Bhalerao, A.; Nakajima, S.; Shiraga, N.; Tamura, S.; Kikinis, R. Tissue classification based on 3D local intensity structures for volume rendering. IEEE Transactions on Visualization and Computer Graphics 2000, 6, 160-180.

40. Li, Q.; Sone, S.; Doi, K. Selective enhancement filters for nodules, vessels, and airway wall in two- and three-dimensional CT scans. Medical Physics 2003, 30, 2040-2051.

41. Hayashi, N.; Masutani, Y.; Masumoto, T.; Mori, H.; Kunimatsu, A.; Abe, O.; Aoki, S.; Ohtomo, K.; Takano, N.; Matsumoto, K. Feasibility of curvature-based enhanced display system for detecting cerebral aneurysms in MR angiography. Magnetic Resonance in Medical Science 2003, 2, 29-36.

42. Suzuki, K.; Zhenghao, S.; Jun, Z. Supervised enhancement of lung nodules by use of a massivetraining artificial neural network (MTANN) in computer-aided diagnosis (CAD). IEEE Conference Proceedings Pattern Recognition (ICPR), Tampa, FL, USA, 2008; pp. 1-4.

43. Giger, M.L.; Chan, H.-P.; Boone, J. Anniversary Paper: History and status of CAD and quantitative image analysis. The role of Medical Physics and American Association of Physicists in Medicine Medical Physics 2008, 35, 5799-5820.

44. Arimura, H.; Katsuragawa, S.; Suzuki, K.; Li, F.; Shiraishi, J.; Sone, S.; Doi, K. Computerized scheme for automated detection of lung nodules in low-dose CT images for lung cancer screening. Academic Radiology 2004, 11, 617-629.

45. Lee, Y.; Hara, T.; Fujita, H.; Itoh, S.; Ishigaki, T. Automated detection of pulmonary nodules in helical CT images based on an improved template-matching technique. IEEE Transactions on Medical Imaging 2001, 20, 595-604.

46. Lancaster, J.L.; Woldorff, M.G.; Parsons, L.M. Automated Talairach atlas labels for functional brain mapping. Human Brain Mapping 2000, 10, 120-131.

47. Toga, A.W.; Thompson, P.M. Maps of the brain. Anaomical Record 2001, 265, 37-53. 
48. Xu, X.W.; Doi, K.; Kobayashi, T.; MacMahon, H.; Giger, M.L. Development of an improved CAD scheme for automated detection of lung nodules in digital chest images. Medical Physics 1997, 24(9), 1395-1403.

49. Vincent, L.; Soile, P. Watersheds in digital spaces: an efficient algorithm based on immersion simulation. IEEE Transactions on Pattern Analysis and Machine Intelligence 1991, 13, 583-598.

50. Kass, M.; Witkin, A.; Terzopulos, D. Snakes: active contour models. International Journal of Computer Vision 1988, 1(4), 321-332.

51. Sethian, J.A. Level set methods and fast marching methods. Evolving interfaces computational geometry, fluid mechanics, computer vision, and materials science. Cambridge Monograph on Applied and Computational Mathematics; Cambridge University Press: Cambridge, UK, 1999.

52. Malladi, R.; Sethian, J.A.; Vemuri, B.C. Shape modeling with front propagation: A level set approach. IEEE Transactions on Pattern Analysis and Machine Intelligence 1995, 17, 158- 175.

53. Zeng, X.; Staib, L.H.; Schultz, R.T.; Duncan, J.S. Segmentation and measurement of the cortex from 3-D MR images using coupled surfaces propagation. IEEE Transactions on Medical Imaging 1999, 18, 100-101.

54. Deng, J.; Tsui, H.T. A fast level set method for segmentation of low contrast noisy biomedical images. Pattern Recognition Letters 2002, 23, 161-169.

55. Magome, T.; Arimura, H.; Kakeda, S.; Yamamoto, D.; Kawata, Y.; Yamashita, Y.; Toyofuku, F.; Higashida, Y.; Ohki, M.; Korogi, Y. Automated method for segmentation of white matter and gray matter regions with multiple sclerosis in 3T MR images. The Institute of Electronics, Information and Communication Engineers (IEICE) Technical Report 2009, 108, 505-506.

56. Li, Q. Improvement of bias and generalizability for computer-aided diagnostic schemes. Computerized Medical Imaging and Graphics 2007, 31, 338-345.

57. Jain, A.K.; Duin, R.P.W.; Mao, J. Statistical pattern recognition: Review. IEEE Transactions on Pattern Analysis and Machine Intelligence 2000, 22, 4-37.

58. Vapnik, V.N. The nature of statistical learning theory -statistics for engineering and information science, 2nd Edition; Springer-Verlag: New York, NY, USA, 1999.

59. Cristianini, N.; Shawe-Taylor J. An introduction to support vector machines: and other kernelbased learning methods; Cambridge University Press: Cambridge, UK, 2000.

60. Yamashita, K.; Yoshiura, T.; Arimura, H.; Mihara, F.; Noguchi, T.; Hiwatashi, A.; Togao, O.; Yamashita, Y.; Shono, T.; Kumazawa, S.; Higashida, Y.; Honda, H. Performance evaluation of radiologists with artificial neural network for differential diagnosis of intra-axial cerebral tumors on MR images. American Journal of Neuroradiology 2008, 29, 1153-1158.

61. Efron, B.; Tibshirani, R. An Introduction to the Bootstrap; Chapman \& Hall/CRC: Boca Raton, FL, USA, 1994.

62. Crum, W.R.; Camara, O.; Hill, D.L.G. Generalized overlap measures for evaluation and validation in medical image analysis. IEEE Transactions on Medical Imaging 2006, 25, 14511461.

63. Kakeda, S.; Moriya, J.; Sato, H.; Aoki, T.; Watanabe, H.; Nakata, H.; Oda, N.; Katsuragawa, S.; Yamamoto, K.; Doi, K. Improved detection of lung nodules with aid of computerized detection method: evaluation of a commercial computer-aided diagnosis system. American Journal of Roentgenology 2004, 182, 505-510. 
64. King, J.T., Jr. Epidemiology of aneurysm subarachnoid hemorrhage. Neuroimaging Clinics of North America 1997, 7, 659-668.

65. Uchiyama, Y.; Ando, H.; Yokoyama, R.; Hara, T.; Fujita, H.; Iwama, T. Computer-aided diagnosis scheme for detection of unruptured intracranial aneurysms in MR angiography. In Proceedings of IEEE Engineering in Medicine and Biology, Shanghai, 17-18 Jan. 2006; pp. 30313034.

66. Kobashi, S.; Kondo, K.; Hata, Y. Computer-aided diagnosis of intracranial aneurysms in MRA images with case-based reasoning. The Institute of Electronics, Information and Communication Engineers (IEICE) transactions on information and systems 2006, E89-D, 340-350.

67. Yamada, T.; Kadekaru, H.; Matsumoto, S. Prevalence of dementia in the older Japanese-Brazilian population. Psychiatry and Clinical Neurosciences 2002, 56, 71-75.

68. Schmidt, R.; Fazekas, F.; Offenbacher, H. Magnetic resonance imaging white matter lesions and cognitive impairment in hypertensive individuals. Archives of Neurology 1991, 48, 417-420.

69. Breteler, M.M.; van Swieten, J.C.; Bots, M.L. Cerebral white matter lesions, vascular risk factors, and cognitive function in a population-based study: The Rotterdam study. Neurology 1994, 44, 1246-1252.

70. DeCarli, C.; Murphy, D.G.M.; Tranh, M. The effect of white matter hyperintensity volume on brain structure, cognitive performance, and cerebral metabolism of glucose in 51 healthy adults. Neurology 1995, 45, 2077-2084.

71. Skoog, I.; Berg, S.; Johansson, B.; Palmertz, B.; Andreasson, L.A. The influence of white matter lesions on neuropsychological functioning in demented and non-demented 85-year-olds. Acta Neurologica Scandinavica 1996, 93, 142-148.

72. Mohamed, F.B.; Vinitski, S.; Gonzalez, C.F.; Faro, S.H.; Lublin, F.A.; Knobler, R.; Gutierrez, J.E. Increased differentiation of intracranial white matter lesions by multispectral 3D-tissue segmentation: preliminary results. Magnetic Resonance Imaging 2001, 19, 207-218.

73. Anbeek, P.; Vincken, K.L.; van Osch, M.J.P.; Bisschops, R.H.C.; van der Grond, J. Automatic segmentation of different-sized white matter lesions by voxel probability estimation. Medical Image Analysis 2004, 8, 205-215.

74. Admiraal-Behloul, F.; van den Heuvel, D.M.J.; Olofsen, H.; van Osch, M.J.P.; van der Grond, J.; van Buchem, M.A.; Reiber, J.H.C. Fully automatic segmentation of white matter hyperintensities in MR images of the elderly. NeuroImage 2005, 28, 607-617.

75. Uchiyama, Y.; Yokoyama, R.; Ando, H. Computer-aided diagnosis scheme for detection of lacunar infarcts on MR images. Academic Radiology 2007, 14, 1554-1561.

76. Zhiqiang, L.; Dinggang, S.; Dengfeng, L. Computer-assisted segmentation of white matter lesions in 3D MR images using support vector machine. Academic Radiology 2008, 15, 300-313.

77. Wen, W.; Sachdev, P. The topography of white matter hyperintensities on brain MRI in healthy 60- to 64-year-old individuals. NeuroImage 2004, 22, 144-154.

78. U.S. National Institute of Health, http://www.nia.nih.gov/Alzheimers/

79. Ministry of Health, Labour and Welfare in Japan, http://www.mhlw.go.jp/index.html

80. Hirata, Y.; Matsuda, H.; Nemoto, K. Voxel-based morphometry to discriminate early Alzheimer's disease from controls. Neuroscience Letter 2005, 382, 269-274. 
81. Li, S.; Shi, F.; Pu, F.; Li, X.; Jiang, T.; Xie, S.; Wang, Y. Hippocampal shape analysis of Alzheimer disease based on machine learning methods. American Journal of Neuroradiology 2007, 28, 1339-1345.

82. Klöppel, S.; Stonnington, C.M.; Chu, C.; Draganski, B.; Scahill, R.I.; Rohrer, J.D., Fox, N.C.; Jack, C.R.; Ashburner, J., Jr.; Frackowiak, R.S.J. Automatic classification of MR scans in Alzheimer's disease. Brain 2008, 131, 681-689.

83. Colliot, O.; Chételat, G.; Chupin, M.; Desgranges, B.; Magnin, B.; Benali, H.; Dubois, B.; Garnero, L.; Eustache, F.; Lehéricy, S. Discrimination between Alzheimer disease, mild cognitive impairment, and normal aging by using automated segmentation of the hippocampus. Radiology 2008, 248, 194-201.

84. Ashburner, J.; Friston, K.J. Voxel-based morphometry - The methods. NeuroImage 2000, 11, 805-821.

85. Campadelli, P.; Casiraghi, E.; Artioli, D. A fully automated method for lung nodule detection from postero-anterior chest radiographs. IEEE Transactions on Medical Imaging 2006, 25, 15881603.

86. Golub, T.R.; Slonim, D.K.; Tamayo, P. Molecular classification of cancer: Class discovery and class prediction by gene expression monitoring. Science 1999, 286, 531-537.

87. Joachims, T. SVM ${ }^{\text {light }}$, Cornell University, Ithaca, NY, USA, http://svmlight.joachims.org/

88. Alfano, B.; Brunetti, A.; Larobina, M.; Quarantelli, M.; Tedeschi, E.; Ciarmiello, A.; Covelli, E.M., Salvatore, M. Automated segmentation and measurement of global white matter lesion volume in patients with multiple sclerosis. Journal of Magnetic Resonance Imaging 2000, 12, 799-807.

89. Boudraa, A.O.; Dehakb, S.M.R.; Zhu, Y.M.; Pachai, C.; Bao, Y.G.; Grimaud, J. Automated segmentation of multiple sclerosis lesions in multispectral MR imaging using fuzzy clustering. Computers in Biology and Medicine 2000, 30, 23-40.

90. Leemput, K.V.; Maes, F.; Vandermeulden, D.; Colchester, A.; Suetens, P. Automated segmentation of multiple sclerosis lesions by model outlier detection. IEEE Transactions on Medical Imaging 2001, 20, 677-688.

91. Zijdenbos, A.P.; Forghani, R.; Evans, A.C. Automatic "Pipeline" analysis of 3-D MRI Data for clinical trials: Application to multiple sclerosis. IEEE Transactions on Medical Imaging 2002, 21, 1280-1291.

92. Khayati, R.; Vafadust, M.; Towhidkhah, F.; Nabavi, S.M. Fully automatic segmentation of multiple sclerosis lesions in brain MR FLAIR images using adaptive mixtures method and markov random field model. Computers in Biology and Medicine 2008, 38, 379-390.

93. Khayati, R.; Vafadust, M.; Towhidkhah, F.; Nabavi, S.M. A novel method for automatic determination of different stages of multiple sclerosis lesions in brain MR FLAIR images. Computerized Medical Imaging and Graphics 2008, 32, 124-133.

94. Abdolmaleki, P.; Mihara, F.; Masuda, K. Neural networks analysis of astrocytic gliomas from MRI appearances. Cancer Letters 1997, 118, 69-78.

95. Kobayashi, T.; Xu, X.W.; MacMahon, H. Effect of a computer-aided diagnosis scheme on radiologists' performance in detection of lung nodules on radiographs. Radiology 1996, 199, 843848 
96. Ashizawa, K.; MacMahon, H.; Ishida, T. Effect of an artificial neural network on radiologists' performance in the differential diagnosis of interstitial lung disease using chest radiographs. American Journal of Roentgenol 1999, 172, 1311-1315.

97. Nakamura, K.; Yoshida, H.; Engelmann, R. Computerized analysis of the likelihood of malignancy in solitary pulmonary nodules with use of artificial neural networks. Radiology 2000, 214, 823-830.

98. Matsuki, Y.; Nakamura, K.; Watanabe, H. Usefulness of an artificial neural network for differentiating benign from malignant pulmonary nodules on high-resolution CT: evaluation with receiver operating characteristic analysis. American Journal of Roentgenology 2002, 178, 657663.

99. Bidiwala, S.; Pittman, T. Neural network classification of pediatric posterior fossa tumors using clinical and imaging data. Pediatric Neurosurgery 2004, 40, 8-15.

100. Abe, H.; Ashizawa, K.; Li, F. Artificial neural networks (ANNs) for differential diagnosis of interstitial lung disease: results of a simulation test with actual clinical cases. Academic Radiology 2004, 11, 29-37.

101. Fukushima, A.; Ashizawa, K.; Yamaguchi, T. Application of an artificial neural network to highresolution CT: usefulness in differential diagnosis of diffuse lung disease. American Journal of Roentgenology 2004, 183, 297-305.

102. Matake, K.; Yoshimitsu, K.; Kumazawa, S. Usefulness of artificial neural network for differential diagnosis of hepatic masses on CT images. Academic Radiology 2006, 13, 951-962.

103. Vijayakumar, C.; Damayanti, G.; Pant, R.; Sreedhar, C.M. Segmentation and grading of brain tumors on apparent diffusion coefficient images using self-organizing maps. Computerized Medical Imaging and Graphics 2007, 31, 473-484.

104. Kitajima, M.; Hirai, T.; Katsuragawa, S.; Okuda, T.; Fukuoka, H.; Sasao A.; Akter, M.; Awai, K.; Nakayama, Y.; Ikeda, R.; Yamashita, Y.; Yano, S.; Kuratsu, J.; Doi, K. Differentiation of common large sellar-suprasellar masses: Effect of artificial neural network on radiologists' diagnosis performance. Academic Radiology 2009, 16, 313-320.

(C) 2009 by the authors; licensee Molecular Diversity Preservation International, Basel, Switzerland. This article is an open-access article distributed under the terms and conditions of the Creative Commons Attribution license (http://creativecommons.org/licenses/by/3.0/). 